\title{
MyWebSteps: Aiding Revisiting with a Visual Web History
}

\author{
Trien V. Do* AND Roy A. Ruddle ${ }^{\dagger}$ \\ School of Computing, University of Leeds, Leeds LS2 9JT, UK
}

\begin{abstract}
This research addresses the general topic of "keeping found things found" by investigating difficulties people encounter when revisiting webpages, and designing and evaluating a novel tool that addresses those difficulties. The research focused on occasional revisits - webpages that people have previously visited on only one day, a week or more ago (i.e., neither frequently nor recently). A three-month logging study was combined with a laboratory experiment to identify ten underlying causes of participants' revisiting failure. Overall, $61 \%$ of the failures occurred when a webpage had originally been accessed via search results, was on a topic a participant often looked at, or was on a known but large website. Then we designed a novel visual Web history tool to address the causes of failure and implemented it as a Firefox add-on. The tool was evaluated in a three-month field study, helped participants succeed on $96 \%$ of revisits, and was also used by some participants to review and reminisce about their "travels" online. Revised versions of the tool have been publicly released as the Firefox add-on MyWebSteps.
\end{abstract}

\section{RESEARCH HIGHLIGHTS}

- Identifying the underlying causes of failure when people revisit webpages.

- Providing evidence how MyWebSteps helped users overcome those causes.

Categories and subject descriptors: Information Search and Retrieval; Information filtering; Selection process; User Interfaces; Graphical user interface; Evaluation/Methodology; Empirical Study; Design; Evaluation

Keywords: Webpage revisiting; visualization; Web history; navigation

Responsible Editorial Board Member: Name

\section{INTRODUCTION}

Based on data from previous studies (e.g., (Weinreich et al., 2006)), an "average" person will make approximately one million visits to webpages during their lifetime. Between one third and one half of visits are return visits to webpages that have been previously seen (Catledge and Pitkow, 1995; Tauscher and Greenberg, 1997; Cockburn and McKenzie, 2001; Weinreich et al., 2006).

*Present address: NDM Experimental Medicine, University of Oxford, Oxford OX3 9DU, UK

†Corresponding author: R.A.Ruddle@leeds.ac.uk
The plethora of techniques that people use to assist revisiting ("keeping found things found") are well documented (Jones et al., 2001), and a number of tools have been developed to support revisiting. These tools vary from a browser's built-in functionality (e.g., back and forward buttons, bookmarks and a history list (Weinreich et al., 2006)), to browser add-ons (e.g., Flipora ${ }^{3}$ ) and research applications (e.g., (Teevan, 2007; Won et al., 2009)). However, people still express frustration at not knowing where to "go" in order to find a webpage again (Bruce et al., 2004; Teevan et al., 2004). The true cost of

${ }^{3}$ www. flipora.com 
revisiting is hard to calculate, but it has been estimated that knowledge workers waste $15 \%$ of their time as a result of difficulties experienced while trying (and often failing) to find information that they know already exists (Feldman, 2004). That wastage accrues from the time spent searching for the information, time spent redoing work after failing to find information, and consequential losses to an organisation.

The present research focuses on webpages that are difficult to find because a user has visited them neither frequently nor recently (e.g., wishing to revisit a particular page that the user visited about a month ago). We hypothesize that users would benefit from a visual Web history for this type of revisiting, because of the role of visual cues in recognition (Kaasten et al., 2002; Won et al., 2009), notwithstanding the fact that the visual history approaches that were investigated in previous research (e.g., (Cockburn and Jones, 1996; Hightower et al., 1998; Mayer and Bederson, 2001)) have not been adopted in mainstream browsers. The present research involved making a detailed study of revisiting failures during a three-month logging study and a laboratory experiment, and then the design and a three-month field evaluation of a novel visual Web history tool (MyWebSteps) that addressed causes of those failures.

The main contributions of the research are: (a) identifying the underlying causes of revisiting failure, and (b) a detailed evaluation that shows how the tool's functionality helped users to overcome those causes during revisiting.

\section{RELATED WORK}

This section is divided into three parts. First, we briefly review previous research into Web navigation as a whole. Then three approaches to revisiting webpages are described. Finally, approaches of presenting a history to users are summarized.

\subsection{Webpage navigation and revisitation}

Since the early days of the Web, logfile-based methods have been used to analyze users' navigation patterns so that browsers and search engines can be improved (Catledge and Pitkow, 1995; Tauscher and Greenberg, 1997; Cockburn and McKenzie, 2001; Weinreich et al., 2006; Obendorf et al., 2007; Adar et al., 2008; Zhang and Zhao, 2011). These studies have also shown that between one third and one half of visits to pages are revisits, which may be classified according to recency and frequency.

Recency is the time that has elapsed since the last visit to a webpage. Previous research found that the vast majority $(72.6 \%)$ of revisits were made within one hour, and progressively fewer within one day (12\%), within one week $(7.8 \%)$ or after more than one week $(7.6 \%)$ (Obendorf et al., 2007). The present study divided recency into two classes, which were "recently" (revisited in $<4$ days) and "not recently" (revisited in $4+$ days), and our primary focus is on supporting the latter.

The frequency with which users visit webpages follows a Zipf distribution, with most pages visited once, some visited twice, and only a very small number visited on a large number of occasions (Breslau et al., 1999). Frequency is generally considered to affect the ease with which users can revisit information (Bruce et al., 2004; Elsweiler and Ruthven, 2007), and this is reflected in the results of a study where participants were asked to revisit a particular website (as opposed to a specific webpage). Participants' success rate varied from $100 \%$ (websites visited every day) to $90 \%$ (one or two visits per year), though it should be noted that the high success rate may have been caused by the fact that a participant's own description of what they did on a given website was used to create a textual cue for the revisit attempt (Elsweiler and Ruthven, 2007). The present study classified a webpage as visited "frequently" if it was visited on two or more days during the period of the study, and "not frequently" if it was only visited on one day.

The length of time that users dwell on webpages varies considerably. Around $50 \%$ of webpages are looked at for 12 seconds or less, $70 \%$ for 30 seconds or less, and only $10 \%$ for more than two minutes (Weinreich et al., 2006). A dwell time of 30 seconds or more on a webpage can be indicative of webpage utility (Fox et al., 2005), and this threshold was used to analyze search trails in Web logs (White and Huang, 2010). The present study used this threshold to select webpages from participants' Web history for the revisiting experiment.

Users often leave browsers running for extended periods of time, so heuristics need to be used to distinguish one navigation session from another. A common approach is to use a time-out (period of user inactivity). Previous work has adopted thresholds such as 25.5 minutes (Catledge and Pitkow, 1995) or 30 minutes (Kelly and Belkin, 2004; Liu et al., 2010; Tyler and Teevan, 2010). The present research used a 25.5 minute threshold.

\subsection{Methods for revisiting webpages}

Revisiting methods may be classified into three categories: (1) using explicit Web history, (2) using automatically recorded Web history, and (3) using search engines. The following sections review these methods, and for further detail the reader is referred to (Mayer, 2009). 


\subsubsection{Using explicit Web history}

An explicit Web history is a collection of webpages that users think that they would need to visit again in the future, so they manually record the location of the webpages. This allows users to revisit webpages at any point in the future.

One way of manually recording the location of a webpage is to use a Web browser's bookmarks/favorites. Each bookmark entry references only a single page, which loses contextual information (e.g., the navigational path to the page and other pages visited in the same session) (Jones et al., 2001). Although people rarely use bookmarks for large numbers of webpages (Catledge and Pitkow, 1995; Jones et al., 2001), they do tend to bookmark particular webpages that contain interesting/important information (Jones et al., 2015). A number of Web applications also provide a bookmarking capability. For example, Delicious ${ }^{4}$ allows users to store, share and discover Web bookmarks (Miller et al., 2010), Pinterest $^{5}$ lets users "pin" a webpage to a "board" and uses the first image in the webpage to represent the webpage, and Evernote ${ }^{6}$ allows users to annotate webpages for later retrieval.

Several tools have attempted to reduce the effort required to maintain bookmarks. HiBo (Kokosis et al., 2005) automatically organizes bookmarks into topical categories using a built-in subject hierarchy, and HyperBK (Staff and Bugeja, 2007) automatically classifies a webpage into an existing bookmark category. These systems partly address the problem of maintenance effort but, to revisit a webpage, users need to figure out to which category the webpage belongs, and the accuracy of categorizing algorithms needs improving (only 61\% of bookmarks were classified correctly with HyperBK).

The explicit Web history method supports revisiting for any combination of recency and frequency. However, a fundamental limitation is that users need to anticipate the webpages that they will wish to revisit and be willing to put in the manual effort that is required to maintain the history.

\subsubsection{Using automatically recorded Web history}

There are a number of entirely automatic methods for recording history. One that is familiar to Web users is links changing color when a page has been visited, which is designed to show pages that users have visited before. However, the color change is often overruled by style sheet settings, and expires after a period of time that depends on users' browser settings. A browser's Back and Forward buttons are also familiar to Web users, and are frequently

\footnotetext{
${ }^{4}$ https: //delicious.com

${ }^{5}$ https://www.pinterest.com

${ }^{6}$ https://evernote.com
}

used to return to pages in the current navigational session. In two well-known studies, backtracking was the second most used navigation method after hyperlinks, and accounted for $14 \%$ of all navigation actions (Catledge and Pitkow, 1995; Weinreich et al., 2006).

Long-term revisiting is supported by a Web browser's history list, which automatically records the URLs of pages that a user visits. Users may search and browse within the history list, with some browsers allowing users to select entries from temporal categories (e.g., today, or last week; Firefox and Internet Explorer) and others providing buttons for navigation (e.g., newest, newer, older, and oldest; Google Chrome). However, the information provided for each entry is typically limited to the visit date/time, page title and URL, and a favicon image for the website.

Google History ${ }^{7}$ improves on a Web browser's history list by adding two new features. First, it provides a calendar so users can easily navigate to different points of time by date, month and year. Second, Google History captures both search queries and the results pages that users clicked on. Some research tools have also provided more sophisticated functionality than a browser history list. For example, xMem (Ceri et al., 2006) categorizes pages into topics by exploiting semantic information from titles and URLs, and CWH (Won et al., 2009) lets users search with metadata and illustrates results with the thumbnail image of each webpage.

Although users rarely access the history list directly (Tauscher and Greenberg, 1997; Jones et al., 2001; Weinreich et al., 2006) the information that it contains underpins URL auto-completion (the drop-down list that is shown in a browser's URL bar), and displays of a person's most frequently visited pages (a grid of thumbnails of webpages in a new blank tab of a browser.)

Today, the functionality of most Web browsers can be expanded with add-ons/extension, including some that have been developed to support revisiting. Flipora is available for Firefox, Chrome and Internet Explorer. It works like the history list of Web browsers but stores the list on a server so that users can access it anywhere. Rather than using a conventional list of text, History Tree (Panasiti, 2009) displays the history of open tabs as a tree. The nodes in each branch are the sequence of webpages that were opened in a given tab, and each node contains a webpage's title and visit time. However, when the browser is closed, all of the history is deleted.

An automatically recorded Web history stores the data needed to revisit any webpage, avoiding the need for users to anticipate future revisits. However, the size of a user's history typically becomes very large over time, so history tools need to provide users with an effective interface

${ }^{7}$ https://history.google.com/history 
for browsing and searching the history, and recognizing specific webpages within it.

\subsubsection{Using search engines}

To revisit webpages, users often adopt a strategy of searching from scratch (Jones et al., 2001). An analysis of one-year's worth of the Web search query logs of 114 anonymous users revealed that as many as $40 \%$ of all queries were re-finding queries (Teevan et al., 2007). However, users often submitted different queries to refind pages than to originally find a page (e.g., changes in terms of word order, stop words, symbols, stemming, pluralization, abbreviations or synonyms). Therefore, a challenge of using search to revisit webpages is that it is difficult for users to remember the exact search queries that they used in the first place (Aula et al., 2005). Even if an identical query is used, recognizing the correct result on which to click and browse further presents other challenges, particularly if the results ranking has changed (Aula et al., 2005; Teevan et al., 2007).

Some tools have been developed to support the search again ('re-search') strategy (Obendorf et al., 2007). One is the Re:Search Engine (Teevan, 2007), which customizes search results of search engines by fetching relevant, previously viewed results from its cache. Another, the Revisit Rack (Morgan and Wilson, 2010), combines a results list with page thumbnails to aid visual recognition. SearchBar (Morris et al., 2008) and Google History list search queries and the search results that were clicked. Although all of these tools provide benefits, none have dealt directly with the case that the page a user wishes to revisit is multiple clicks on from a given results page. In other words, the tools provide little assistance to users who adopt an "orienteering" strategy of issuing a query to reach the locality of a given webpage, and then using cues and contextual knowledge to make a series of further steps to the desired page (Teevan et al., 2004).

\subsection{Methods for presenting a Web history}

The most common method for presenting history information is a textual list that users need to scan to identify a given webpage. This approach is adopted by browsers for URL auto-completion, bookmarks/favorites and history lists, as well as research tools such as the Re:Search Engine (Teevan, 2007) and SearchBar (Morris et al., 2008).

A study of how people recognize previously visited webpages revealed that thumbnails are a more effective cue than a page title or URL (Kaasten et al., 2002). Since then thumbnails have been exploited to support searching and revisiting in many other studies (Woodruff et al., 2001, 2002; Dziadosz and Chandrasekar, 2002; Teevan et al., 2009; Aula et al., 2010; Morgan and Wilson, 2010; Jiao et al., 2010; Loumakis et al., 2011; Badesh and Blustein, 2012). Browsers also use thumbnails in a limited fashion to support revisiting. For example, Google Chrome and Firefox present a grid of thumbnails of the most frequently visited webpages whenever users open a new blank tab.

A number of research projects have developed tools to visualize web histories. Some of that research adopted the Data Mountain concept (Robertson et al., 1998) to exploit users' spatial memory via $2 \mathrm{D}$ and $3 \mathrm{D}$ arrangements of thumbnails. Despite the lack of significant performance differences between the 2D and 3D interfaces, participants preferred the 3D interface (Cockburn and Mckenzie, 2001).

Other research adopted network visualization methods to present users' navigation. Some of these showed the whole of a user's navigation (e.g., WebNet (Cockburn and Jones, 1996), WebPath (Frecon and Smith, 1998) and Nestor Navigation (Eklund et al., 1999)), but the drawback of this is that the network becomes non-planar and cluttered. An alternative is to display a subset of the user's navigation as a tree (e.g., PadPrints (Hightower et al., 1998), Domain Tree Browser (Gandhi et al., 2000) and SessionGraphs (Mayer and Bederson, 2001)). Evaluations that compared PadPrints and SessionGraphs with Netscape Navigator showed that these tools significantly reduced the number of webpages and time required for revisits. Participants also rated their degree of satisfaction higher with these tools. The Domain Tree Browser addressed scalability by using three panes for presentation (the domain pane, tree pane, and webpage pane), and representing each domain by a separate tree.

Network visualizations have not yet been incorporated into the mainstream web browsers. Two reasons may be: (a) concerns about the scalability of such visualizations during extended usage, and (b) lack of evidence of the benefits for users. Both of these are addressed in our field evaluation (see Section 5).

\section{THE CAUSES OF REVISITING FAILURE}

This part of the present research aimed to answer three questions: (i) in long-term revisiting, does recency affect success, (ii) does the method of cueing a participant's memory about a webpage affect revisiting success, and (iii) what are the underlying causes of any failures that occur during revisiting?

A Firefox add-on was developed to capture an individual's Web navigation, storing the data within the user profile on their computer. Each participant used the add-on for three months, and took part in three 1-hour sessions of a controlled laboratory experiment. During each session, participants were asked to revisit "target" webpages that they had previously visited on only one 
day, either $7 \pm 3$ days previously (termed "1 week" in the remainder of this paper) or $28 \pm 3$ days previously (termed "1 month"). Each target was described textually, in a manner designed to simulate the scenario where a participant had a vague memory of information they wished to find again ("I remember something about X ... but where was it?"), and cued in one of the following ways: (a) anchor text the participant had clicked on, (b) a thumbnail image of the page, (c) a page that preceded the target on the participant's browsing path, or (d) no supplementary cue.

\subsection{Method}

\subsubsection{Participants}

Twelve participants ( 6 females), with a mean age of 26.2 years $(S D=3.9)$, completed the study. The participants were undergraduate and postgraduate students (two studying history, one biology, seven computer science, and two computing \& management). Participants were recruited via tear-off flyers. All the participants gave their informed consent and were paid an honorarium of $£ 18$ for their participation. The study was approved by our University Research Ethics Committee.

\subsubsection{Logging participants' day-to-day Web navigation}

A Mozilla Firefox add-on was developed to automatically capture an individual's Web navigation, by storing webpage information (thumbnail, URL, title) and navigational information (visit time, URL and ID of referrer, anchor text used to access the page, and dwell time) in the individual's personal file space. To save space, a webpage's thumbnail was only stored again if the page had changed since the last visit. For privacy reasons, the add-on did not store any information about https webpages, allowed users to specify that certain websites or pages should not be recorded, and allowed users to turn recording on/off by clicking a button. The add-on also provided an editor, which let users view and delete entries in their Web navigation history.

For each participant, the add-on was installed on one computer. For nine participants this was the only computer (a laptop) that they used during the period of the study, whereas the other three participants used two computers so the add-on was installed on the computer that participants used most for the Web. Between them, participants used the add-on on Windows, Mac and Linux computers.

\subsubsection{Revisiting experiment}

The experiment adopted a within-participants design with factors of recency ( 1 week vs. 1 month) and cue (none vs. anchor text vs. thumbnail vs. path). The choice of cues was informed by previous research (Kaasten et al., 2002;
Fujii, 2008; Li and Zhao, 2009; Dai and Davison, 2010; Koolen and Kamps, 2010).

Each participant was asked to revisit 48 "target" webpages, divided between three sessions that took place at intervals of approximately one week. Each session involved two targets for each of the eight combinations of recency and cue. The first session was typically during the 7 th week of using the tool, but the timing depended on participants using the Web enough to generate sufficient target pages. All of the revisiting sessions were conducted on participants' own computers so they had access to their usual working environment (e.g., bookmarks and Web browser's history). Before each session, participants sent the logfile generated by the add-on to the experimenter so that target webpages could be determined, and the descriptions and cues could be generated.

A computer program was written to select target webpages from each participant's logfile. A target had to meet three criteria: (1) it had been visited on only one day during the whole period of the study, (2) it had been visited either 1 week or 1 month before the revisiting session in which it was used, and (3) the participant must have dwelled on that page for at least 30 seconds to increase the likelihood that the target page was of interest to a participant and would be memorable. Of course, the third criterion could have been satisfied for other reasons (e.g., the participant got distracted). The experimenter also checked that the page still existed.

Each webpage was described by text extracted from: (a) two pairs of two consecutive words chosen randomly from a target page's <title> tag (one pair from each half; if a title contained less than four words then all of them were used), and (b) two keywords extracted from the page's content by the Alchemy Web service ${ }^{8}$. The four pairs/keywords were then sorted randomly. However, these keywords were then reviewed manually because sometimes the keywords might: (a) not be appropriately extracted (e.g., a meaningless title (Won et al., 2009)), or (b) not distinguish a particular webpage, because a participant visited several webpages about the same topic. Pilot testing showed that this method of describing the pages was sufficiently precise for participants to identify the target (subsequently, they were successful in $80 \%$ of the experiment's trials; see Section 3.2), without trivializing the revisiting task in the experimental setting (participants failed to complete $20 \%$ of the revisits).

In each session, a participant first clicked the "Load Target Pages" button in a browser dialog window to load a list of 16 target pages chosen by the computer programme (see Fig. 1), then searched/browsed at their own pace until they had attempted to revisit all these targets, which were presented in a randomly ordered list.

${ }^{8}$ http://www.alchemyapi.com/api/keyword/urls.html 
When the participant clicked on an item in the list, the corresponding target page's description and cueing information (anchor text, thumbnail, etc.) were displayed in the window, and remained visible while the participant tried to revisit the target. A "Verify" button was also displayed in the window (the button "Open Target Page in Browser" in Fig. 1).

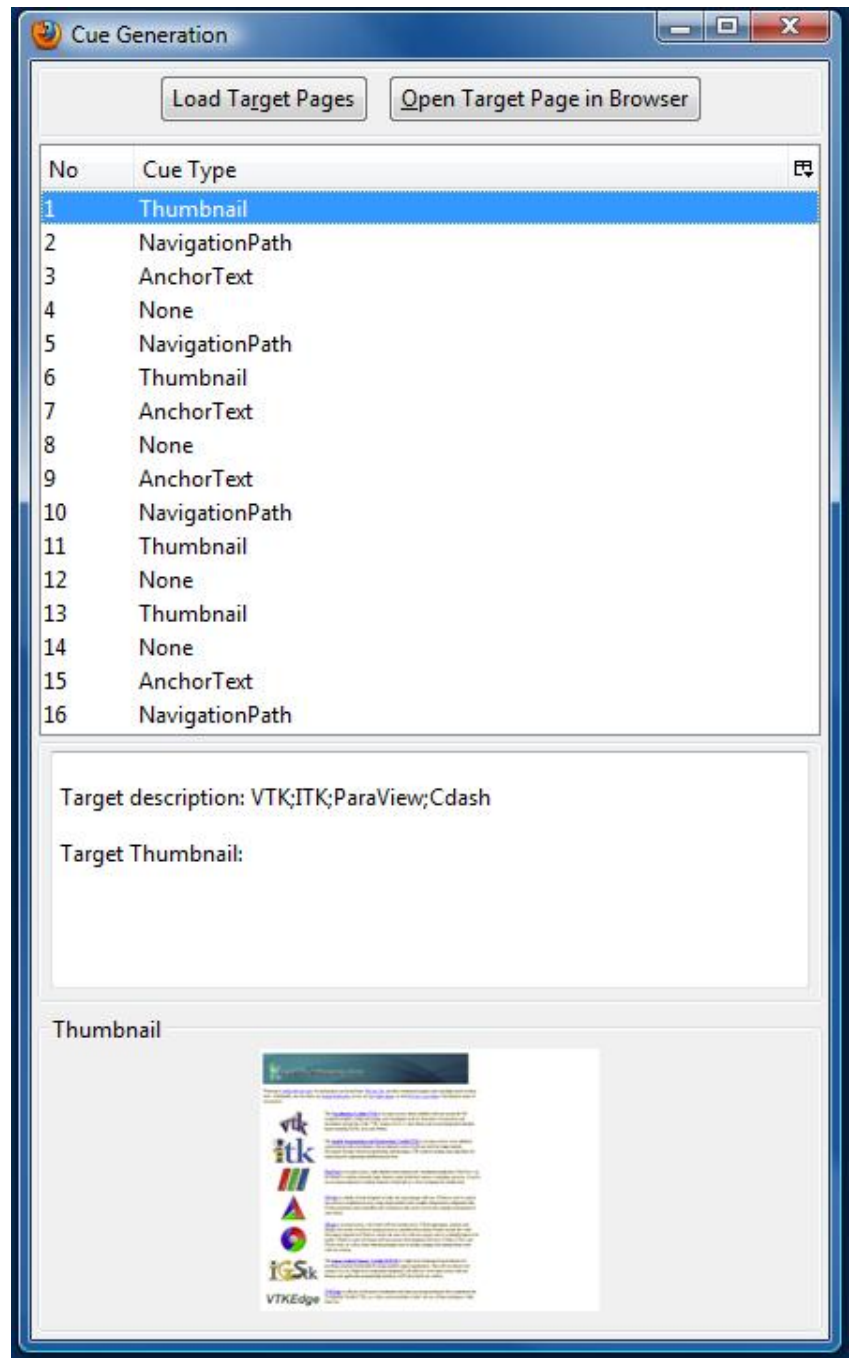

Figure 1. The dialog that presented a list of target pages to a participant.

For each target, the procedure was as follows. First, the participant selected the item in the list, and read the description and cue. Next, the participant was asked whether they recalled the page concerned, recalled the topic, how they previously found the page, and what they were looking for on that occasion. After that, the participant tried to find the target using any method they wished. The participant was asked to "think aloud" while looking for the target, and if they were silent for more than about 10 seconds then the experimenter reminded them to keep talking. Once the participant thought that they had found the target they clicked on the "Verify" button to open the target to verify whether the revisit had been successful. If a target page was not found within 3 minutes of the item being selected in the dialog window list then the trial was terminated as unsuccessful (Gwizdka and Spence, 2007).

All three sessions were videoed using a digital camcorder and logged using the add-on for subsequent analysis. The camcorder was placed on a tripod behind participants and captured their monitor, keyboard and mouse. The videos also recorded what participants said about target pages, including their memory for them and any difficulties that were expressed while thinking aloud during revisiting.

\subsection{Results}

The day-to-day logfile data allowed comparison of our participants' Web navigation activity with that of previous studies (see Table 1). Broadly speaking, our participants' activity was similar to that of the most recent of the listed studies (Zhang and Zhao, 2011), and differences with earlier studies are likely to have been influenced by changes in browser interfaces (e.g., the introduction of multiple tabs) and the wholesale evolution of the Web.

During the study, the participants visited 154-722 different websites $(M=512)$ and 733-6163 different webpages $(M=5335)$. An average of $84.1 \%$ of the webpages were only visited once by a given participant. The other $15.9 \%$ of webpages were visited multiple times, together accounting for the $36 \%$ recurrence rate. These revisited webpages were subdivided into four groups according to visit frequency and recency (see Table 2). If a webpage was visited on more than one day then we classified it as visited "frequently", and if a webpage was ever revisited in less than 4 days then we classified it as visited "recently". Almost one fifth of the revisited pages were in the "neither recently nor frequently" category, which was the focus of the present research. The following sections report participants' performance and strategy in the revisiting, and analyze the unsuccessful trials in detail to identify underlying causes of revisiting failure.

\subsubsection{Revisiting performance and strategy}

Each participant was asked to revisit six targets for each combination of recency ( 1 week vs. 1 month) and cue (anchor text vs. thumbnail vs. path vs. none), and all of the targets were in the not recently/not frequently category of Table 2 . In total, 576 trials (12 participants $\mathrm{x}$ 6 targets $\times 2$ recencies $\times 4$ cues) were performed. The data were checked to ensure that they were normally distributed and satisfied the assumption of sphericity. A 
Table 1. Comparison of the present study with previous Web navigation studies. The recurrence rate is the percentage of page visits that were revisits.

\begin{tabular}{|l|l|l|l|l|l|l|}
\hline Measure & $\begin{array}{l}\text { (Catledge } \\
\text { and Pitkow, } \\
1995)\end{array}$ & $\begin{array}{l}\text { (Tauscher } \\
\text { and Green- } \\
\text { berg, 1997) }\end{array}$ & $\begin{array}{l}\text { (Cockburn } \\
\text { and McKen- } \\
\text { zie, 2001) }\end{array}$ & $\begin{array}{l}\text { (Obendorf et } \\
\text { al., 2007) }\end{array}$ & $\begin{array}{l}\text { (Zhang and } \\
\text { Zhao, 2011) }\end{array}$ & $\begin{array}{l}\text { Present } \\
\text { study }\end{array}$ \\
\hline $\begin{array}{l}\text { Number of partici- } \\
\text { pants }\end{array}$ & 107 & 23 & 17 & 25 & 20 & 12 \\
\hline Duration (days) & 21 & $35-42$ & 119 & $52-195$ & 31 & $50-97$ \\
\hline Recurrence rate & $61 \%$ & $58 \%$ & $81 \%$ & $46 \%$ & $39 \%$ & $36 \%$ \\
\hline $\begin{array}{l}\text { No. of URL visits } \\
\text { per day }\end{array}$ & 14 & 21 & 90 & 80 & 86 \\
\hline
\end{tabular}

Table 2. Frequency and recency breakdown of the $15.9 \%$ of webpages that were visited multiple times.

\begin{tabular}{|l|l|l|}
\hline Frequency $/$ recency & Frequently & Not frequently \\
\hline Recently $(<4$ days $)$ & $2.1 \%$ & $10.0 \%$ \\
\hline Not recently $(4+$ days $)$ & $0.9 \%$ & $2.9 \%$ \\
\hline
\end{tabular}

repeated measures analysis of variance showed no effect of recency $(\mathrm{F}(1,11)=0.37, \mathrm{p}=.55)$, cue $(\mathrm{F}(3,33)=2.45$, $\mathrm{p}=.08)$, or a significant interaction $(\mathrm{F}(3,33)=0.38, \mathrm{p}$ $=.77)$. Overall, participants failed to revisit an average of 1.2 out of 6 targets (20\%) for each combination of recency and cue (see Fig. 2).

The failure rate increased as participants' memory for the targets decreased. In the majority $(79 \%)$ of trials participants stated that they specifically recalled the target, but still had a failure rate of $13 \%$. In $16 \%$ of trials participants remembered the general topic but had a failure rate of $46 \%$, and in $5 \%$ of trials participants stated that they did not remember anything about a target, but the failure rate was only $57 \%$ because participants sometimes found it by searching/browsing and then recognizing the target page.

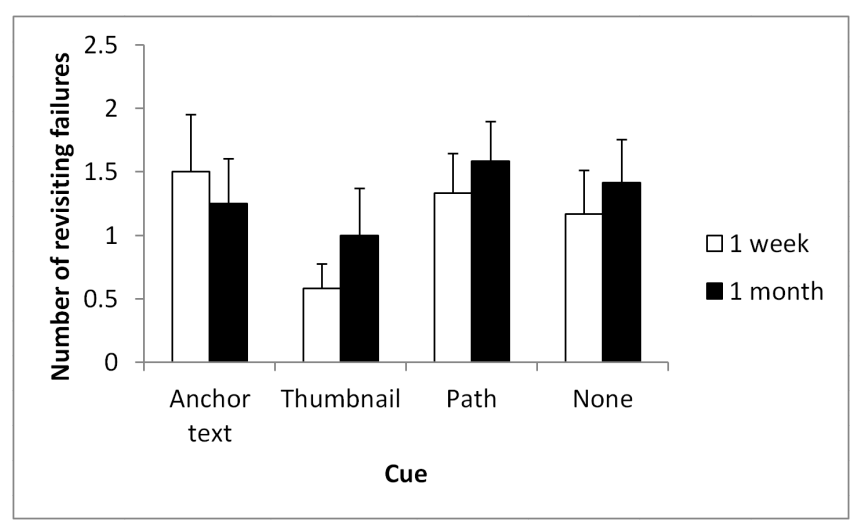

Figure 2. Mean number of targets that participants failed to revisit for each combination of recency and cue. Error bars show the standard error of the mean.
The video data were used to identify participants' revisiting strategy in each trial (see Table 3). Two of the strategies involved participants searching and browsing the Web for the target (re-search and re-trace, respectively (Obendorf et al., 2007)). Two other strategies involved use of data stored by the browser (search in history; bookmark), and participants sometimes used a mixture of strategies or did not try.

\subsubsection{Causes of revisiting failure}

The failure trials were analyzed in detail to establish the underlying causes of failure. First, videos of all 118 failure trials were transcribed to text and classified by the first author using the emergent encoding approach (Haney et al., 1998). Then, data source triangulation (Erlandson et al., 1993) was used to validate the analysis, by checking what participants said in the videos against the logfiles. For example, when a participant said she remembered the target page belonged to a search session, the related logfile containing that webpage was examined to review that original search session. The analysis confirmed that what participants said in the videos was consistent with the logfiles and revealed 10 underlying causes for the revisiting failures (see Table 4). The logfiles were also used to distinguish between pages that a participant had previously visited (i.e., at any point during the study) vs. new pages (pages that the participant visited for the first time during the experiment). The remainder of this section illustrates the causes.

The two most commons causes were topic and search results (see Table 4). The topic cause occurred when a participant visited pages from a variety of websites over an extended period of time, e.g., following a sport 
Table 3. Number of trials and failure rate for participants' revisiting strategies.

\begin{tabular}{|l|l|l|l|}
\hline Strategy & Description & $\begin{array}{l}\text { Number of } \\
\text { trials }\end{array}$ & $\begin{array}{l}\text { Failure } \\
\text { rate for } \\
\text { strategy }\end{array}$ \\
\hline Re-search & $\begin{array}{l}\text { Search from scratch by typing keywords into a search } \\
\text { engine }\end{array}$ & 289 & $23 \%$ \\
\hline Search in history & $\begin{array}{l}\text { Type keywords into address bar or history dialog to } \\
\text { see suggestions from browser history }\end{array}$ & 166 & $7 \%$ \\
\hline Re-trace & Type in URL and then browse & 84 & $26 \%$ \\
\hline Bookmark & Select bookmark, because target is thought to be one & 3 & $67 \%$ \\
\hline Mixture & Two or more of above methods & 26 & $38 \%$ \\
\hline Did not try & Participant did not attempt to revisit target & 8 & $100 \%$ \\
\hline Total & & $\mathbf{5 7 6}$ & - \\
\hline
\end{tabular}

Table 4. Number of failure trials for each underlying cause.

\begin{tabular}{|l|l|}
\hline Cause of failure & Number of trials \\
\hline Topic & 29 \\
\hline Search results & 28 \\
\hline Site found but not specific page & 14 \\
\hline Deleted link & 8 \\
\hline Hidden information & 8 \\
\hline Faceted search & 7 \\
\hline Inappropriate page title & 6 \\
\hline Links from email, forums and social networks & 3 \\
\hline Multi-page thread & 2 \\
\hline Do not remember & 13 \\
\hline Total & $\mathbf{1 1 8}$ \\
\hline
\end{tabular}

event that lasted several days/weeks or doing research for an assignment. When asked to revisit a particular page, participants typically gave up after revisiting several pages and said something like:

"I've visited a few webpages about $X X X$ when I did $Y Y Y$, I'm not sure which page contains the specific content mentioned in the description. It might be page A".

The search results cause occurred when participants had originally used a search engine to find the target. For example, a participant explained:

"I remember when I was watching an art program on $T V$ the other day I especially liked a painting. I used Google to search for it. It was tricky as I had to use different keywords and clicked on quite many links. I'm not sure if I can find it again".

The other eight causes were less common. Site found but not specific page occurred when participants correctly recalled the website that a target page belonged to (the first step of an orienteering approach (Teevan et al., $2004)$ ), but could not find the page again. In one example of this cause a participant said:
"Oh! This page was a call for research proposals of EPSRC". Then he went to the EPSRC website and started browsing but could not find the page, and explained: "Last time I browsed within this website a lot and saw this page. But to be honest, I can't remember how I reached it".

The deleted link cause occurred when participants remembered where they had previously found the target, but the link to it had disappeared. Link rot is a wellknown problem (Ntoulas et al., 2004), and is particularly common on news websites, which are frequently updated. One participant said:

"Every day, I read news on BBC. This article was in the front page, but now hmm it is not there anymore. I don't know how to find it again".

Some webpages only initially show certain information and users need to interact (e.g., clicking links, tabs or images) to view details. This led to the hidden information cause, which occurred when participants revisited the correct page, but did not recognize it because they did not interact to reach to the information they sought. 
The faceted search cause happened when participants navigated with faceted search (Tunkelang, 2009) on an accommodation, shopping or similar website. On these websites users often search and then make filtering decisions, which participants found challenging to repeat to find a target. Such websites are database-driven, with URLs that are designed to be machine- rather than human-readable, and page titles that only partially state the current filtering, so browser history functionality is of limited help.

The inappropriate page title cause primarily occurred when participants adopted a search in history strategy, which relies on words contained in a title or URL. People's memory for URLs and titles is generally poor (Won et al., 2009). In addition, search in history tends to break down if a page has a generic title or one that is clearly inappropriate.

Participants sometimes initially visited pages via links from email, forums $\&$ social networks. Revisiting failures occurred if the emails were deleted or the posts were no longer available, because the path that participants wished to follow to re-trace their steps was broken. People sometimes avoid this by saving URLs in other documents (Bruce et al., 2004).

Forums are widely used nowadays to discuss ideas, share knowledge, etc. Forum members contribute posts in threads, and the multi-page thread cause occurred because all of a thread's posts had the same title, so it was difficult for participants to find a specific post.

Lastly, participants sometimes expressed their frustration of knowing a target webpage must be somewhere but they do not remember anything that would allow them to revisit it.

\subsection{Discussion}

Previous studies of revisiting may be divided into three categories. In the first, participants both visit and then revisit information within a controlled, laboratory experiment setting (Hightower et al., 1998; Robertson et al., 1998; Wexelblat and Maes, 1999; Mayer and Bederson, 2001; Ceri et al., 2006). The second is based on logfile data that are recorded during everyday web navigation (e.g. (Catledge and Pitkow, 1995; Tauscher and Greenberg, 1997; Cockburn and McKenzie, 2001; Weinreich et al., 2006)), meaning that a study is naturalistic but lacks the control and follow-up that are possible in a laboratory setting. Our causes of revisiting failure study falls into the third category, which combines naturalistic and laboratory elements to ask participants to return to information they have previously visited during everyday web navigation. Few previous studies have adopted that approach, but exceptions include (Jones et al., 2003; Bruce et al., 2004; Elsweiler and Ruthven, 2007; Elsweiler et al., 2011).

The modest number of participants who took part in the study means that a certain amount of caution should be exercised when interpreting the results for the four cues. However, the number of revisiting failures did not differ significantly for pages visited one week vs. one month previously, which indicates that a time delay of a week may be sufficient for researchers to study longterm revisiting phenomena. This would be useful when tools designed to assist revisiting are to be evaluated. The failure rate was higher than the $10 \%$ reported by Bruce et al. (2004), which is likely to be because the present study focused on webpages that were visited neither frequently nor recently, and is in line with suggestions that it is more difficult to find "cold" information than "warm" or "hot" information (Elsweiler and Ruthven, 2007).

Participants' revisiting strategies were broadly similar to those identified in previous research (Bruce et al., 2004; Obendorf et al., 2007), with re-search, search in history and re-trace adopted on $74 \%$ of occasions. However, surprisingly few participants knew about the functionality that today's browsers provide for searching within history, and this strategy's low failure rate shows that it would be worthwhile educating users about that functionality.

A key aim of our study was to establish a set of causes for revisiting failure. In the remainder of this section we relate some of those causes to previous research that, in general, aimed to develop methods to make revisiting easier.

Three types of circumstance were equally common for the topic cause. One type was when participants only visited new pages and would clearly have benefited from being provided with a set of pages about the topic that they had previously visited (e.g., by a system such as xMem (Ceri et al., 2006) or manually querying the browser history). In the second, participants failed at the last step of an orienteering approach (Teevan et al., 2004), visiting a page(s) that was linked to the target but not the target itself. The third occurred when participants visited a page(s) that had previously been visited during the same session as the target, and would have benefited from an approach similar to SessionGraphs (Mayer and Bederson, 2001).

Sometimes the search results cause was aligned with well-known problems such as changes of results' ranking (Aula et al., 2005; Teevan et al., 2007), incorrect recall of previous queries (Aula et al., 2005), and ineffective browsing (Obendorf et al., 2007). Once a participant remembered the query that she had previously used but the results list had changed so the target page was not returned, and on eight occasions participants used a different query so the target page was not listed. 
Other failures were a consequence of what participants did once the search results were listed. Ten failures occurred when participants did not click on the correct result, even though the link was colored to indicate that the page had previously been visited. Nine failures occurred when participants clicked on the correct result, but did not continue to the target page.

For the site found but not specific page cause, participants visited almost as many new pages as those they had previously visited (45\% vs. $55 \%$ ). Current Web browsers typically organize a person's history according to when a webpage was visited (Today, Yesterday, Last 7 days, etc.), and then within that allow the entries to be sorted by criteria such as website (e.g., the 'view by site' and 'more from this site' functionality of Internet Explorer and Google Chrome, respectively). However, users would benefit if that functionality was made more visible, rather than being buried within a history menu that users rarely access. This would also help to address problems caused by faceted search.

Finally, recruiting participants is always a challenge in this type of research, because the study lasted for a long period (3 months of logfile capture, and three 1-hour revisiting sessions) and involved sharing personal Web usage data with researchers. The number of participants that we used is similar to some previous influential studies (Jones et al., 2001; Mayer and Bederson, 2001; Teevan et al., 2004; Won et al., 2009) but, of course, limits to the extent to which the results may be generalized. However, the participants' background strengthens rather than diminishes the findings because they demonstrate that even young, well-educated users who are familiar with the Web have difficulties with revisiting.

\section{DESIGN OF A NEW TOOL FOR AIDING WEB REVISITING}

The following section describes the design of MyWebSteps, a new visual Web history tool that aims to make revisiting faster and more successful. The tool addresses four key causes of revisiting failure (topic, search results, site found but not specific page, and deleted link), which together accounted for $79 \%$ of the failures in the revisiting experiment. Technical details about the tool's implementation are described in the online supplementary data.

A common problem that arose in the above four causes is that there were often several candidate webpages and a user could not identify the precise webpage that they wished to revisit until they saw it. This led to three general requirements:

1) Users need to be able to filter their Web history to select a small subset of possible webpages.
2) The tool needs to present that subset of webpages in a visual manner, so users can recognize and choose the page they want.

3) To support cases where users find it difficult to find that page, the tool needs to allow users to navigate that subset of their Web history.

The tool was designed in three iterations. First, three users (including one who was a specialist in humancomputer interaction) provided feedback about a paper prototype. Next, the same users provided feedback about a working version of the tool following one month of field usage. Finally, minor changes were made and incorporated into the version that was used for the field evaluation (see Section 5). The following sections describe the tool's design and how that was expected to address the causes of revisiting failure.

\subsection{Design}

MyWebSteps' overall layout has some similarities with Microsoft Outlook, which is familiar to millions of users, and has three components: Global Navigation, Results View, and Toolbar (see Fig. 3).

\subsubsection{Global Navigation}

As discussed in Section 2.3, scalability is one of the main unknowns about using a visualization to present a Web history. Even in one year, an average user visits thousands of different webpages (Weinreich et al., 2006). Although it is possible to present that quantity of webpages at one time (e.g., using a fish-eye view (Furnas, 1986)) it would be difficult for users to recognize a specific webpage. MyWebSteps addresses scalability by enabling users to select subsets of their Web history from the Global Navigation component. This component provides a calendar and allows users to filter their history by switching between tabs for domains, searches, and sessions. On the calendar, selecting a date displays all of the webpages/search queries/sessions that were visited on that day, and highlights the date itself in red. The calendar uses a five-shade heatmap to indicate the number of webpage visits or search queries on each day. A mouseover text displays the number of visits/search queries on a given day.

By default, the domains tab lists all of the domains that a user has visited. However, if a date is selected then only domains visited on that date are listed. If the user selects a domain then all of the pages they have visited in that domain (and, optionally, on the selected date) are shown in the Results View.

The other tabs work in a similar manner. The searches tab lists all of the user's Google search queries by default, or those for a given date if a user selects one on the calendar. Selecting a query allows users to see the results 


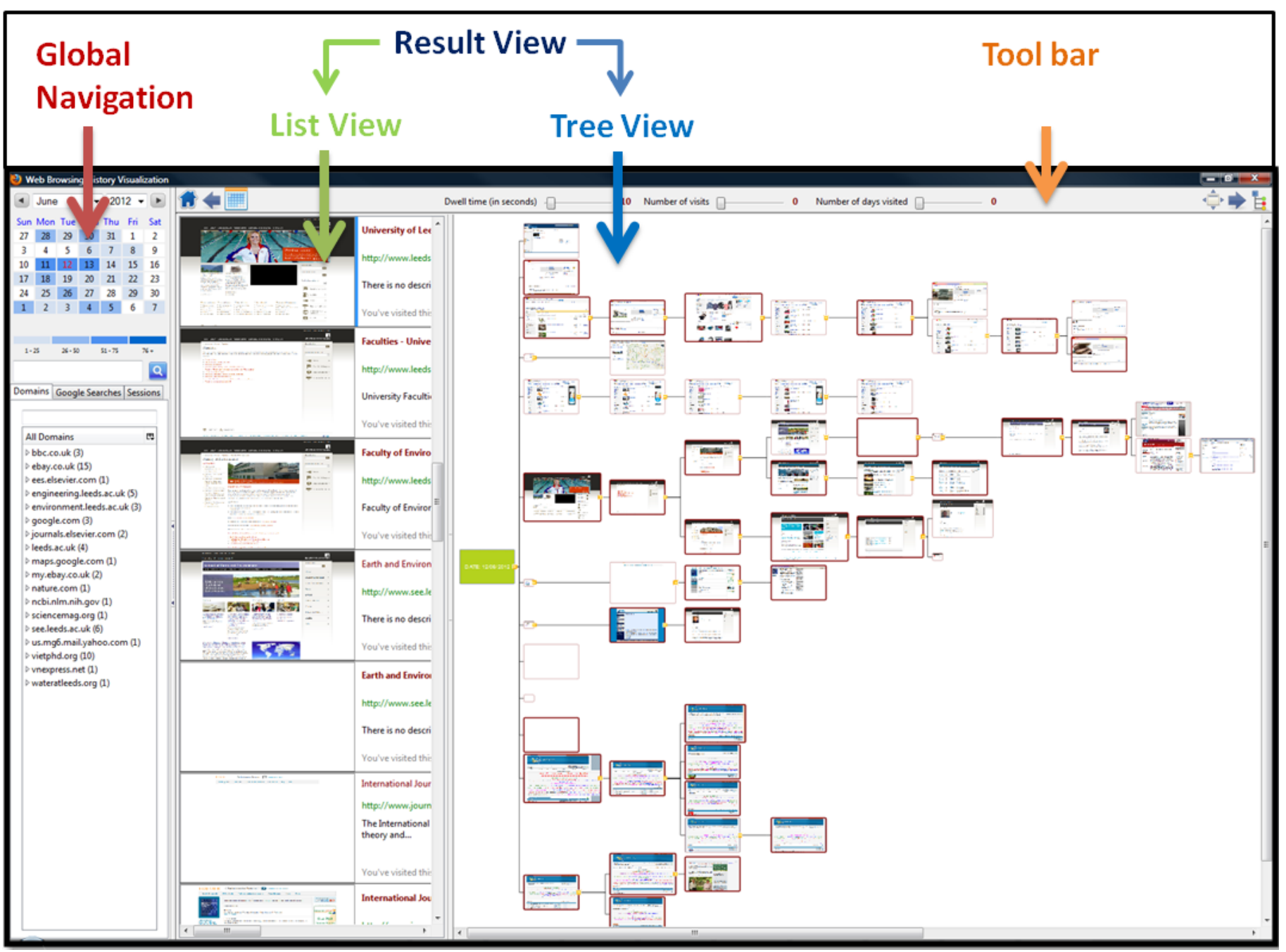

Figure 3. MyWebSteps comprising the Global Navigation component with a heat map calendar and a tab view (left), the list and tree view parts of the Results View (right), and the Toolbar (top).

page that was generated for that query and all of the webpages that were browsed to from any of the results. The sessions tab uses the 25.5 minute timeout criterion to divide a user's navigation into sessions, optionally just listing the sessions that occurred on a given date.

Inevitably, over time the list of domains/searches/sessions will become long. To address this scalability issue, a user may type text into a textbox below the tabs to only list domains/searches/sessions that match. Complementing this, a search box above the tabs allows the user to search for all pages that have given text in their title or description.

\subsubsection{Toolbar}

The toolbar provides buttons to reset the filters and calendar, let users undo/redo their actions in the tool's interface, fit the tree results view to the screen, display the domains/searches/sessions for every day in the calendar's current month, and switch between showing either only the list view in the Results View or both the list and tree views. The toolbar also contains sliders to filter webpages in the Results View by dwell time, number of visits (frequency), and number of days visited (e.g., a webpage might have been visited several times but only on one or two days). These filters remove pages from the list view, but in the tree view pages are shrunk so that the tree structure (see Fig. 3) does not change.

\subsubsection{Results View}

This component displays the webpages that a user has selected with the Global Navigation and Toolbar controls, so the user can recognize the webpage that they wish to revisit. The left hand part of the view shows a list of the pages, with the thumbnail, title, URL and description of 
each. The right hand part shows the thumbnails as a tree, linked according to the user's navigation. The list may be scrolled, and the tree panned and zoomed.

In a Web history, some pages are visited multiple times. However, for scalability our list and tree views only display each page once, providing information about visit frequency via text (list view) and thumbnail size (tree view), as well as allowing users to filter by frequency (see Section 4.1.2).

The list view (see Fig. 4) orders pages by when they were first visited, with the most recent page at the top. This temporally groups pages and provides users with contextual information because one page may act as a cue for another that was visited at a similar time. Taking advantage of ideas used in Google search results and a number of research tools (Cockburn and Jones, 1996; Mayer and Bederson, 2001; Won et al., 2009), each page's title, URL, description, visit frequency ("You've visited this webpage $\mathrm{X}$ times") and visit recency ("Last visited ...") are provided. This textual information is enriched by a thumbnail that conveys the layout of a page and has proved to be useful for users' recognition (Kaasten et al., 2002; Won et al., 2009). As a trade-off between showing recognizable detail and the number of thumbnails that may be displayed at the same time, a thumbnail height of 148 pixels is used (Kaasten et al., 2002; Won et al., 2009).

Pages of interest (dwell time $\geq 30$ seconds) are represented with bold titles to distinguish them with other pages. When a user chooses to display only the list view, a high-resolution image of the currently page is also shown (see Fig. 4), changing instantly when the user selects another page in the list.

The tree view (see Fig. 5) uses a tree structure that approximates how a user navigated between the pages that are shown. This approximation is a trade-off between showing a simple layout that is fast to compute vs. the difficult to read, non-planar graph that would be produced if every navigation action was shown as a link. Other history tools have also come to the same conclusion and used trees (Domel, 1995; Gandhi et al., 2000; Mayer and Bederson, 2001).

The tree view is rendered horizontally, from left to right. Thumbnails are used for the tree nodes because that is useful for user recognition. The default size of a node is the same as the thumbnail's size used in the list view. A thicker border depicts pages of interest (dwell time $\geq 30$ seconds). Similar to WebNet (Cockburn and Jones, 1996) and SessionGraphs (Mayer and Bederson, 2001), a node's size encodes the frequency of visits to the page.

The tree's root node is a text box that states the filters that are currently in use (e.g., date and domain being viewed). The thumbnail of every page that satisfies the filter criteria is shown as a node, and the tree edges show how a user navigated to each page. For example, if the user clicks on a hyperlink in webpage $\mathrm{A}$ to go to page $\mathrm{B}$ then there is an edge from $\mathrm{A}$ to $\mathrm{B}$, and if page $\mathrm{C}$ is visited by direct entry (e.g., typing in the URL or a bookmark) then there is an edge from the root node to C. The details about how a tree is created are described in the online supplementary data.

When the mouse is over a node on the tree view, the corresponding item on the list view is highlighted by a blue bar. In both the list and the tree view users can either double click on a page's thumbnail to open the page in a new tab of the browser or may right click on it to open a dialog which shows detailed information about the page (see Fig. 6). An image that is $75 \%$ of the resolution of the page is used to display the page's previous (i.e., historical) content. The dialog also provides additional options such as opening the current version of the page and viewing every webpage the user visited in the same domain, during the same session, about the same topic, or navigated to from the page.

\subsection{Addressing the causes of revisiting failure}

This section describes scenarios related to the main causes of failure, and an explanation of how MyWebSteps' design should help a user successfully revisit desired pages. Each scenario is based on our practical experience with the tool.

\subsubsection{Topic}

This scenario concerns a user who spent two weeks researching bikes before deciding to buy one. To order it he needed to go back to its webpage, but all of the bike pages had similar content and he could not identify the correct page until he saw it. The solution is to just type "bike" into the search box to see all the webpages he had visited that were about bikes, and scroll the list to find the bike he wants.

\subsubsection{Search results}

In this scenario a user remembers that he searched for information about the best places to work a while ago and wants to go back to a particular result. However, he has used the tool for years, so it has logged many search queries. The solution is this. He thinks his original query was something like 'best place', so he starts to type into the searches tab dialog. After typing one character ('b') 127 matching queries are shown, and typing the second character ('e') reduces this to 25 queries. By the time he has typed 'best' there are only seven matching queries in the list, and he sees the query 'best places to work'. He clicks on it, looks at the tree view (see Fig. 7) to see the pages he subsequently visited, and recognizes that the top right page is the one he wants. It is not one of the search results - it was one to which he navigated from a result. The search box provides two key advantages. First, 


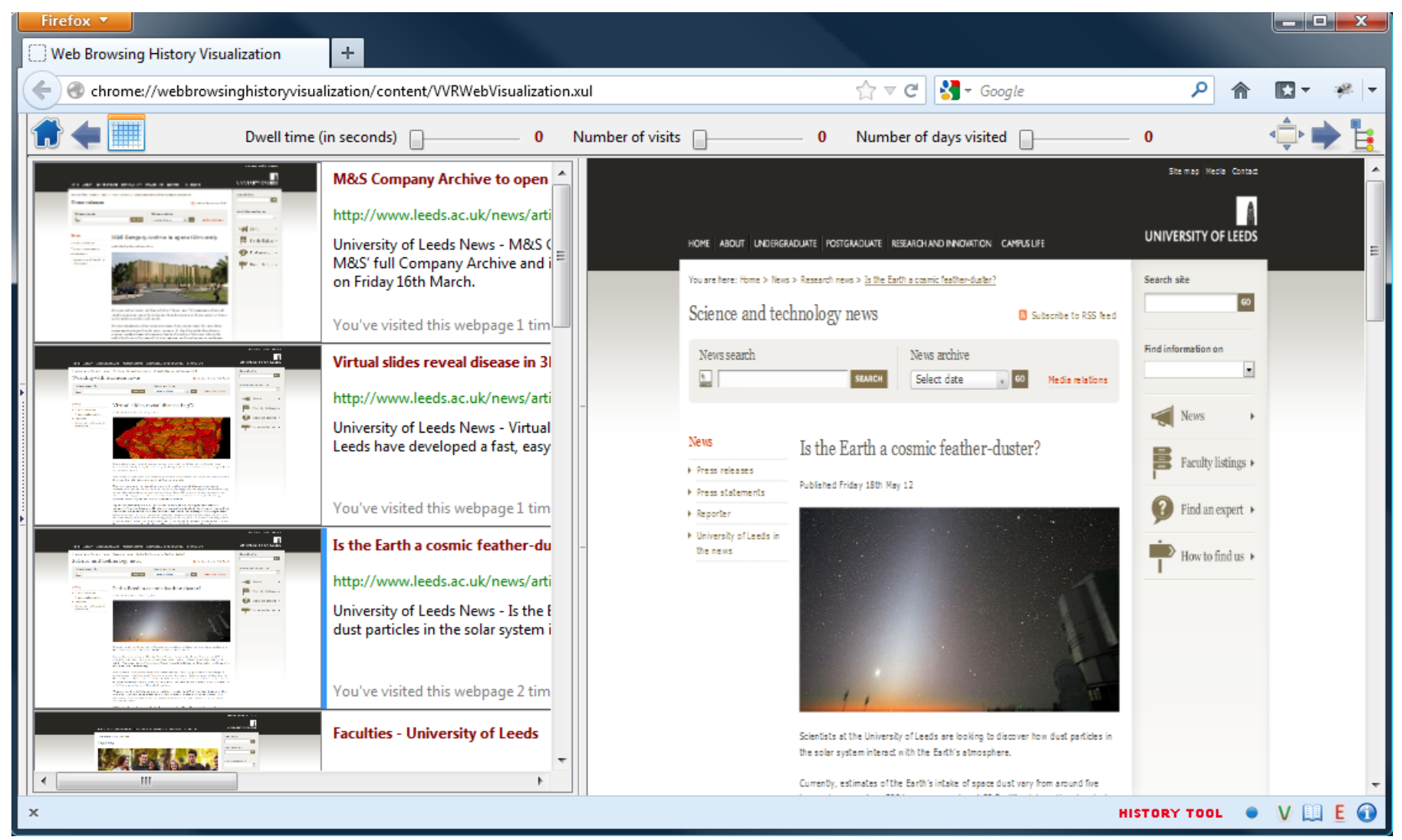

Figure 4. The 'list view only' mode displays a high-resolution image of the currently selected page in the space normally occupied by the tree view.

it dynamically filters the queries as a user types. Second, it allows the user to see all of the webpages that they navigated to from a given query.

\subsubsection{Site found but not specific page}

In this scenario a user remembers that she read news on her university website and browsed to information about the 'Turing centenary conference'. A couple of days later, she talks to her friend who also mentions this conference. When she gets back to her desk, she wants to look again at the conference information, but does not remember much about it except the name 'Turing'. The solution is this. First she types the university's domain into the textbox of the domain tab, which shows her that she has visited pages on the main university site and in several sub-domains. Of those, she makes a guess that the information should be in either the School of Computing or Mathematics. She clicks on the computing sub-domain but does not recognize the desired webpage. Then she tries the mathematics subdomain and finds all of the webpages about the conference.

\subsubsection{Deleted link}

In this scenario a user remembers that she read news about a study to discover how dust particles in the solar system interact with the Earth's atmosphere. Several days later, she wants to read that information again, but new stories have replaced old ones on the site's homepage, as is particularly common with media organizations. As a solution, she opens the tool and goes to the site's homepage (she knows its domain). Right clicking on that node, she selects the option to 'view all webpages visited from this webpage', and switches to the 'list view only' mode to see thumbnails and high-resolution images of each page. Scrolling through the list view, she then recognizes the page she is looking for.

\subsubsection{Other causes}

Although the tool was primarily designed to address the aforementioned causes of failure, it also addresses others. For example, if the underlying causes is hidden information, faceted search, or multi-page thread then a user could select a specific domain in the domains tab and then recognize the desired webpage from the thumbnails. For the 'links from email, forums \& and social networks' 


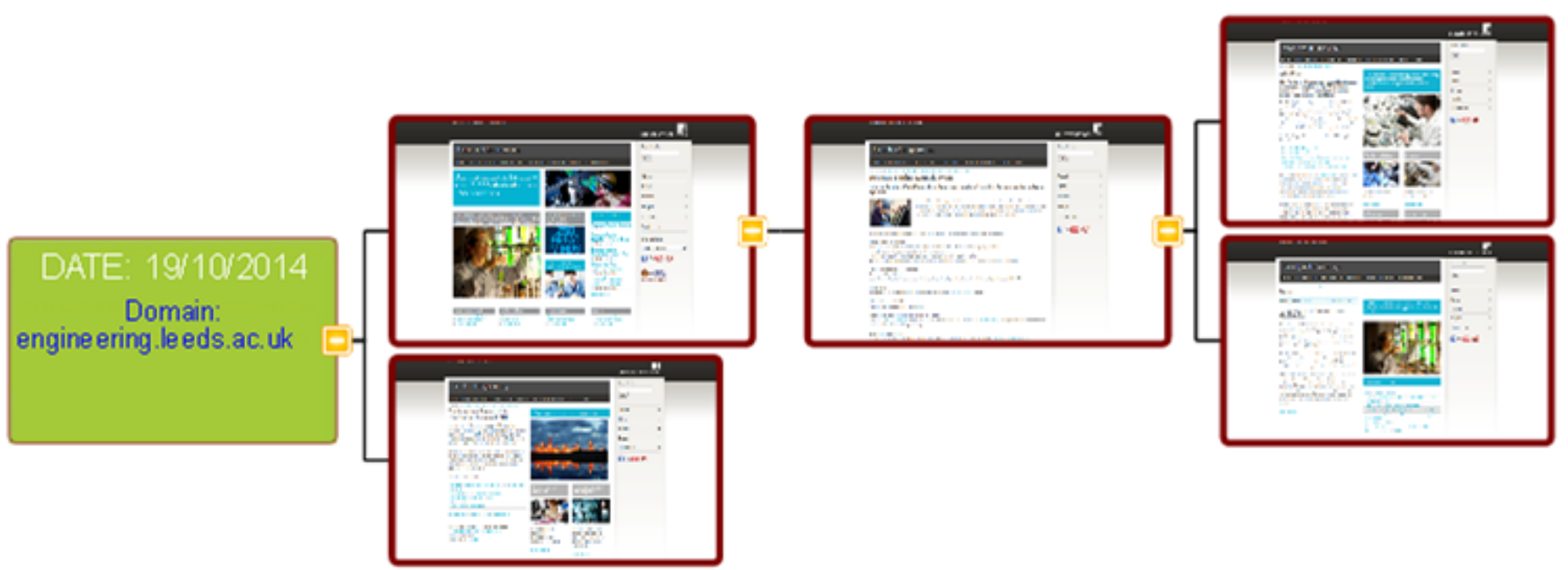

Figure 5. The tree view, showing the root node (left), and two pages reached by direct entry (connected to the root), and three pages reached via hyperlinks from the first direct entry page.

cause, a user may right-click on any page of the relevant forum etc, select 'View all external webpages visited from this domain', and then identify the specific page from those that the tool displays.

\section{FIELD EVALUATION}

The overall goal of this evaluation was to investigate how MyWebSteps assisted revisiting. That goal was subdivided into the following research questions: (a) For which classes of revisiting did participants use MyWebSteps (see Section 5.2.1), (b) How did usage of MyWebSteps relate to the causes of revisiting failure (see Section 5.2.2), and (c) What interface functionality is important for efficient revisiting (see Sections 5.2.3 \& 5.2.4).

The evaluation was a three-month field study, during which the tool recorded participants' everyday browsing and searching. The tool also provided a diary in which participants added comments about revisits to webpages both with and without the tool. At the end of the study, feedback was gathered via semi-structured interviews.

\subsection{Method}

\subsubsection{Participants}

Nineteen participants ( 5 females), with a mean age of 27 years $(S D=7)$, completed the study. Two participants were academic staff in the School of Computing, two were researchers in Computing, three were software engineers, one was a manager, and the rest were $\mathrm{PhD}$ students (one studying in Earth \& Environment, one in Biology, and nine in Computing). Four of them had taken part in the study described in Section 3. Another five participants withdrew from the study, and their data are not reported. The study was approved by our University Research Ethics Committee. All the participants gave their informed consent.

\subsubsection{Materials}

The design of MyWebSteps was as described in Section 4. It recorded participants' web navigation, and provided a diary form that was displayed automatically when a participant opened a webpage using MyWebSteps, revisited a webpage, or clicked on a "diary" icon. The form asked questions about which URL a participant was trying to revisit, when, how and how often they had visited it, how they tried to revisit it, the difficulty of doing so, and whether they were successful. An "any other comments" box was also provided (see Fig. 8). Participants were asked to answer the questions in as much detail as possible.

Three participants only used their laptop during the field study. The rest used more than one device to access the Web (one at home, the other at work, and maybe a smart phone or tablet). In this case, the tool was installed on one computer chosen by these participants. Another participant installed the tool on both of his computers, sending two logfiles that were merged for analysis.

\subsubsection{Procedure}

At the start of the study, participants were sent the MyWebSteps add-on, complete with an online user manual. The participants installed the tool, on completion of which a quick start guide was displayed. A week later, a follow-up email was sent to each participant to ensure that they had no problem with installing and using the tool. Every two weeks during the three months of the study an 
Webpage's Details and Options

Title: Weather Headlines - weather.com

URL: http://www.weather.com/weather/tenday/Dublin+Ireland+EIXX0014

Description: Get the latest weather news and headlines from weather.com.

Dwell time: 33.0 seconds. Frequency: 1 . No. of Days: 1

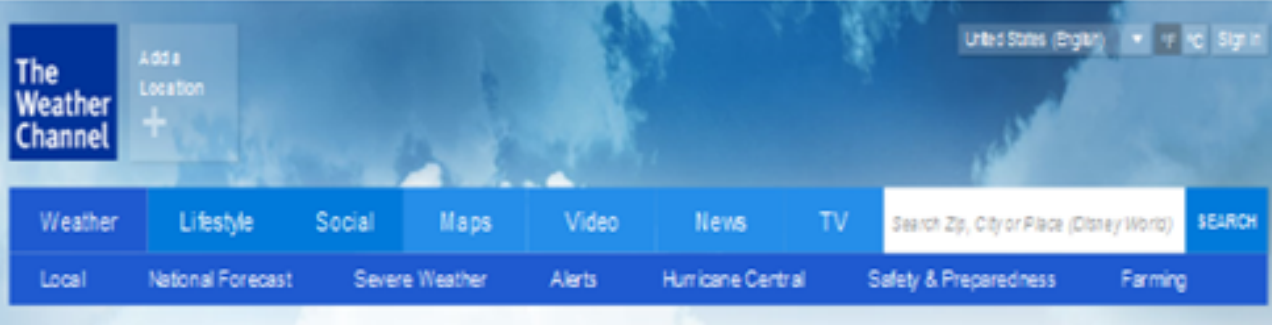

Dublin, Ireland Weather $\dot{B}$

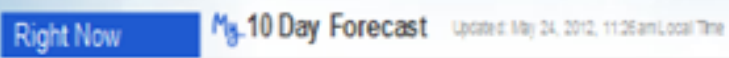

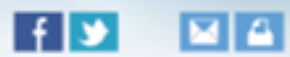

Recommended Videos

- Open this webpage in Firefox

View all webpages visited in the same domain

View all webpages visited from this webpage

View all external webpages visited from this domain

View all webpages on the same day/session

View all webpages visited about the same topic

OK

Cancel

Figure 6. The dialog that was opened by right clicking on a node in the tree view, and displays a webpage's details and navigation options.

email was sent to remind participants to fill in the diary form for revisits.

At the end of the study, each participant was instructed about how to send the logfile back to the researchers. The logfile was then briefly reviewed for a follow-up semistructured interview that, depending on the participant's preference, was conducted face-to-face or via Skype. The interview questions were: (1) Did you read the user manual (yes/no), (2) How would you rate the ease of use of the tool (5 point Likert scale, from Difficult to Easy), (3) Did you notice the information encoded in the colors of the heat map calendar, bold title/border of the nodes, thumbnail size, and tree reconstruction (yes/no in each case), (4) How would you rate your satisfaction with the tool (5 point Likert scale, from Not satisfied to Very satisfied), (5) Will you keep using the tool (yes/no), (6) What was the reason you didn't fill in the diary form (free text), (7) Did you report all cases that you could not find a wanted webpage with the tool (yes/no), and (8) Do you have any difficulties/suggestions?

\subsection{Results}

Participants visited an average of 56 URLs per day, with a recurrence rate of $28 \%$. Responding to questions posed during the interview, participants indicated that the tool was easy to use (average rating of 3.8 on a 5 point Likert scale), a high level of satisfaction ( 4.1 out of 5 ), and that $84 \%$ of participants wanted to keep using the tool after the evaluation.

Analysis of the logfiles showed that each participant used MyWebSteps for an average of 16 sessions $(S D=$ 


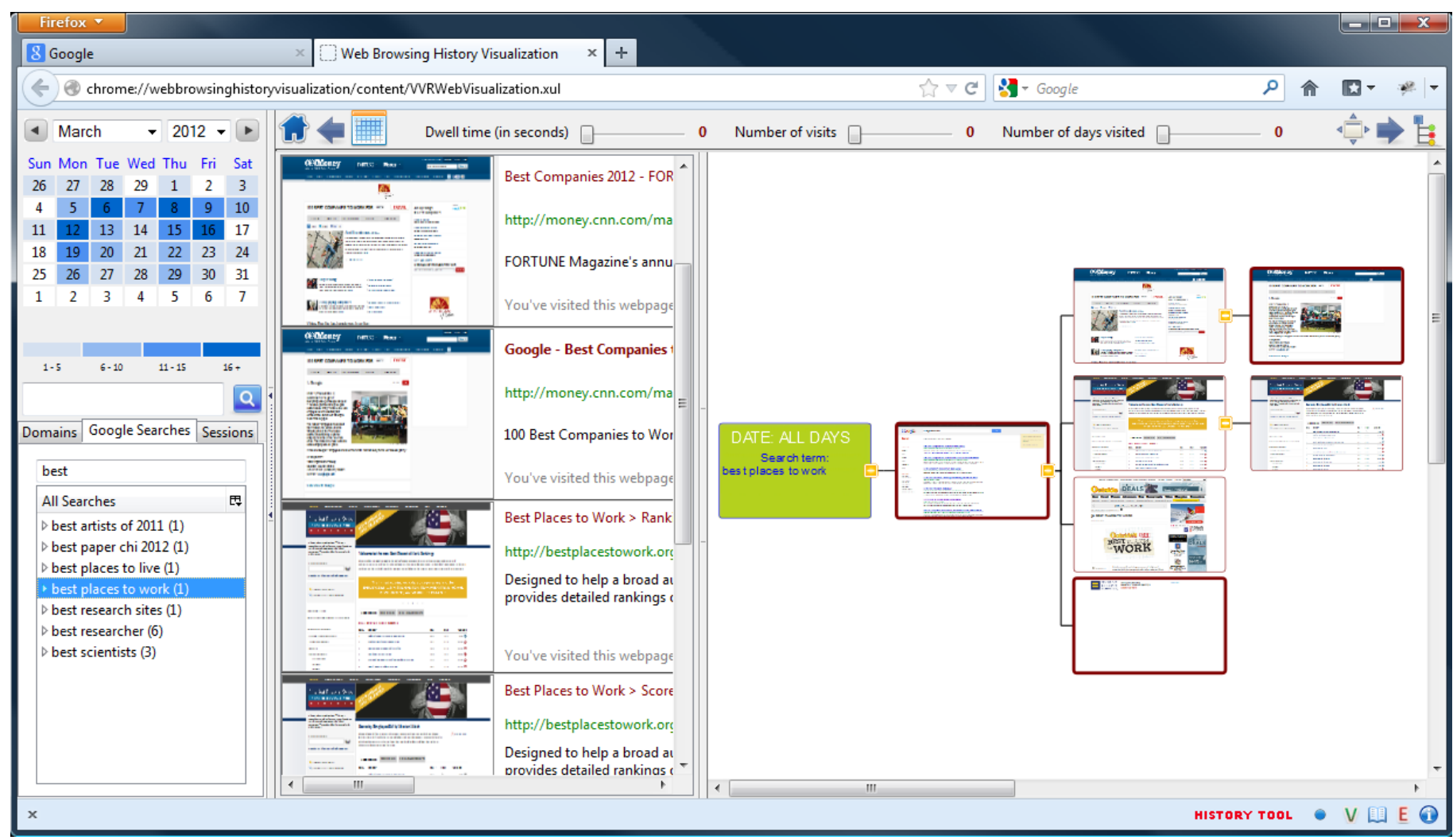

Figure 7. The search results scenario, showing the filtered list of searches and, for a specific search, the search results (linked to the tree's root node), four results that were clicked on, and two pages that were navigated to from a result (the user wants to revisit the top right page).

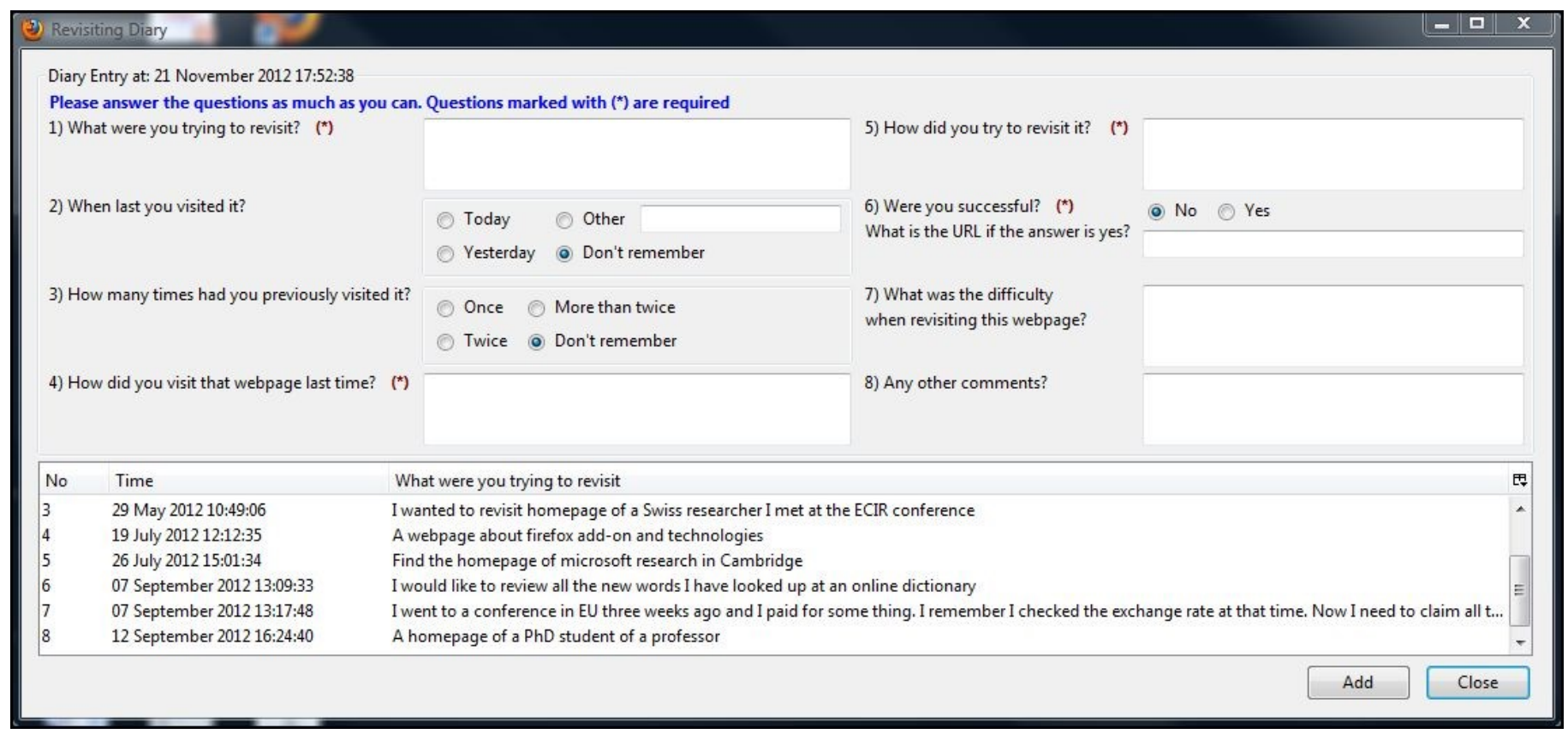

Figure 8. The diary dialog. 
13) during the field study, with a session ending when the tool was closed or after 25.5 minutes of inactivity. These sessions had three distinct purposes: exploring the tool's functionality (22\% of occasions; characterized by participants clicking on various interface controls in the tool without opening many webpages), revisiting a webpage $(48 \%)$, and reviewing browsing history $(30 \%)$.

The following sections report how participants used the tool for revisiting, covering recency and frequency, the causes of failure, the methods used for revisiting, and the views and interface functionality. Then we briefly describe how the tool was used in reviewing sessions.

\subsubsection{Revisiting recency/frequency}

Revisiting was defined as when a participant either revisited a webpage or used the diary form to indicate that they had not found the webpage for which they were looking. Analysis of the logfiles indicated that the recency/frequency pattern for browser revisiting was similar to the study described in Section 3 (see Tables 2 vs. 5). Participants primarily used browser functionality to return to pages that had been visited recently. However, as expected, MyWebSteps was primarily used for pages that had been visited neither recently nor frequently $(50.4 \%)$. In terms of quantity, participants performed more revisits with a browser than MyWebSteps $(M=972$ vs. 8). However the latter included non-trivial revisits that participants considered were substantially easier with our tool.

In total, 111 diary entries involved the use of MyWebSteps, and participants indicated that they were successful on $96 \%$ of those occasions. On $32 \%$ of occasions participants explicitly stated that they chose the tool over conventional browser functionality because it made revisiting easier, and on $20 \%$ of occasions participants switched from directly entering a URL to using the tool to open the webpage concerned. On another 34 occasions participants used the tool to revisit a webpage without filling in a diary entry.

Considering all 145 occasions when MyWebSteps was used, the logfile data showed that revisiting took an average of 15 seconds $(S D=17)$ from participants opening the tool to the desired webpage being displayed in the web browser. Revisiting took an average of 3.3 interaction steps $(S D=1.3)$, including a first step to open the tool.

\subsubsection{Revisiting vs. the causes of failure}

Participants' 111 diary entries were analyzed as follows to relate revisiting carried out with the tool to the underlying causes of revisiting failure. First, participants' diary entries for how they had previously visited a webpage and how they tried to revisit it were checked against the logfiles. This allowed uncertainties to be corrected (e.g., where a participant stated "not sure" or "search possibly"), and revealed only one inconsistency where a participant thought that he had visited a webpage via a search session but the logfile showed that he had browsed to this webpage when he was reading news. Second, adopting the content analysis method with $a$ priori coding (Weber, 1990), diary entries were classified using the previously identified underlying causes of failure. For example, entries were classified as the Search results cause when participants stated that they had previously used a search engine to visit a webpage but preferred to use the tool rather than search again to revisit the webpage. When diary entries could not be classified into any causes of failure, new categories were created (see below). Data source triangulation (Erlandson et al., 1993) was performed by checking the diary entries against the logfile data for the tool usage and a participant's everyday navigation. Third, both stability (intra-coder reliability) and reproducibility (inter-coder reliability) were checked. For stability, the first author repeated the coding after one week, with $94 \%$ agreement. For reproducibility, the second author independently coded the diary entries, with $75 \%$ agreement. Cohen's Kappa (Cohen, 1960) was 0.61, indicating satisfactory reliability (threshold $=0.6$ ), and the coding differences were resolved by discussion.

The number of diary entries that were classified under each cause of revisiting failure is shown in Table 6, including three new categories (direct entry, only remember appearance, and old version). Examples of the comments from participants' diaries for seven of the causes are:

\section{Topic:}

"I have been reading a lot on this topic for my dissertation over the summer. During the writing up, I often need to go back to some webpages to add references. I found the search functionality fantastic. I just needed to type the topic then recognized which webpage contained the information I needed"

Search results:

"I knew I could have found the webpages with Google search again but I preferred the tool because it was much easier and faster."

Site found but not specific page:

"Quite useful to go directly to a part of a large website (rather than browsing via main page)"

\section{Inappropriate page title:}

"I often type some characters in the address bar to see suggestions from the history, however in this case I could remember what the page was about but not what the title was."

\section{Direct entry:}

This occurred when participants had previously visited a webpage by typing in an URL or using a bookmark. They had no difficulty revisiting the webpage, but the 
Table 5. Frequency and recency breakdown of webpages that were visited multiple times with conventional browser functionality vs. MyWebSteps.

\begin{tabular}{|l|l|l|l|l|}
\hline \multirow{2}{*}{ Recency/frequency } & \multicolumn{2}{|l|}{ Revisiting with normal browser } & \multicolumn{2}{c|}{ Revisiting with MyWebSteps } \\
\cline { 2 - 5 } & Frequently & Not frequently & Frequently & Not frequently \\
\hline Recently $(<4$ days $)$ & $12.6 \%$ & $58.4 \%$ & $13.1 \%$ & $26.2 \%$ \\
\hline Not recently $(4+$ days $)$ & $7.2 \%$ & $21.8 \%$ & $10.3 \%$ & $50.4 \%$ \\
\hline Total & \multicolumn{2}{|c|}{$\mathbf{1 0 0 \%}$} & \multicolumn{2}{|}{$\mathbf{1 0 0 \%}$} \\
\hline
\end{tabular}

tool made revisiting much easier, e.g., "... if I clicked on an item in the domain list, then this gave me thumbnails from which I could select a particular one. I found this very useful because I often knew the domain name, but was unsure where the page I wanted lay within the domain. It gave me easy access deep within a domain, without needing to keep lots of bookmarks."

\section{Only remember appearance:}

This occurred when participants could recall what a webpage looked like but little else about it, e.g., "I closed the tab then realized I wanted to look at the page again. It would have been a nightmare to find the page again by searching, because I'd looked at many images to find one that was suitable for an illustration. The list view made it easy to revisit - just recognize the image I wanted from near the bottom of the list"

Old version:

This occurred when a participant wanted to see the content of a webpage on a particular day in the past, which the tool preserved in the webpage's thumbnail, providing a personal "Internet Archive"9. The participant commented: "The tool is more than just a revisiting tool. It is also the archive of my web history. The other day I needed to claim my conference expenses. The tool helped me get the exact foreign exchange rate in the past".

The four failures (see Table 6) each occurred for a different reason. One occurred when a participant tried to find a webpage within a website he knew well. He noticed that no webpages from that website had been recorded, and this was because the website used the https protocol. For privacy, the tool does not record https webpages (see Section 3.1.2). The second involved a website where all of the pages looked very similar and a participant selected the correct domain but picked the wrong thumbnail, though he reported that he was still able to navigate to the desired page from there. The third was when a participant tried to find a scientific paper again on Web of Knowledge. However, the participant's logfile had no entries for Web of Knowledge, and the follow-up interview indicated that the participant may have accessed that paper from a different computer. The fourth failure occurred when a participant

\footnotetext{
${ }^{9}$ http://archive.org/web/
}

could not remember the search term she had used to previously find a webpage. She tried to filter domains, searched with a keyword, browsed through some dates and opened some webpages but she still could not find the one she was looking for.

\subsubsection{Methods used to revisit webpages}

During revisiting with MyWebSteps, participants filtered their history data in a variety of ways (see Table 6). This section compares how participants interacted during revisiting with a baseline provided by conventional browser functionality (the versions of three popular browsers that were current at the time of writing; Firefox version 38, Internet Explorer version 11, and Google Chrome version 43).

Like our tool, Firefox, Internet Explorer and Chrome all allow users to filter history by entering free text. Users typically perform this by typing into a browser's address bar, but filtering may also be performed via the browser's history menu. However, the browsers typically only provide the URL of each page, and sometimes title and visit date/time. Our tool provides much richer information, including the thumbnail to aid recognition, and of course this also applies to the other filtering methods that are described below.

Filtering to only show pages visited today involves a similar amount of interaction with our tool and the three browsers (2-3 clicks; assuming the menu bar is already visible). However, there are substantial differences for filtering by domain. Firefox does not provide that capability, and the functionality is not obvious in either Internet Explorer (users need to view their history and then select a drop down menu to choose 'View by site') or Chrome (users need to find a webpage from the domain and then realize that clicking an arrow icon will reveal a drop down menu that allows 'More from site' to be selected). By contrast, our tool displays a list of domains in the Global Navigation panel, so that users may select a domain with a single click.

For filtering by date, the browsers adopt two distinct approaches. Firefox and Internet Explorer allow users to select intervals of time (e.g., this month, or 2 weeks ago), but this lack of precision substantially increases the 
Table 6. Classification of diary entries against the underlying causes of failure, and the filtering that participants applied to successfully revisit webpages with MyWebSteps..

\begin{tabular}{|c|c|c|c|c|c|c|c|c|}
\hline \multirow{2}{*}{ Cause } & \multirow{2}{*}{$\begin{array}{l}\text { Total No. } \\
\text { of diary } \\
\text { entries }\end{array}$} & \multicolumn{7}{|c|}{ Number of diary entries with given filter applied } \\
\hline & & Text & Today & Domain & Date & $\begin{array}{l}\text { Date/ } \\
\text { domain }\end{array}$ & Searches & Other \\
\hline Topic & 10 & 3 & 2 & 2 & 2 & 0 & 0 & 1 \\
\hline Search results & 55 & 21 & 11 & 9 & 6 & 5 & 2 & 1 \\
\hline $\begin{array}{l}\text { Site found but not specific } \\
\text { page }\end{array}$ & 4 & 1 & 1 & 1 & 0 & 1 & 0 & 0 \\
\hline Deleted link & 3 & 1 & 0 & 0 & 1 & 1 & 0 & 0 \\
\hline Faceted search & 4 & 0 & 0 & 2 & 1 & 0 & 0 & 1 \\
\hline Hidden information & 0 & 0 & 0 & 0 & 0 & 0 & 0 & 0 \\
\hline Inappropriate page title & 1 & 0 & 0 & 0 & 0 & 1 & 0 & 0 \\
\hline $\begin{array}{l}\text { Links from email, forums and } \\
\text { social networks }\end{array}$ & 5 & 0 & 0 & 2 & 2 & 1 & 0 & 0 \\
\hline Multi-page thread & 0 & 0 & 0 & 0 & 0 & 0 & 0 & 0 \\
\hline Direct entry & 21 & 0 & 3 & 9 & 8 & 1 & 0 & 0 \\
\hline Only remember appearance & 3 & 0 & 1 & 0 & 0 & 1 & 0 & 1 \\
\hline Old version & 1 & 0 & 0 & 0 & 0 & 0 & 1 & 0 \\
\hline Failed & 4 & 0 & 0 & 2 & 1 & 0 & 1 & 0 \\
\hline Sub-total & 111 & 26 & 18 & 27 & 21 & 11 & 4 & 4 \\
\hline No diary and entry & 34 & 1 & 14 & 8 & 2 & 5 & 3 & 1 \\
\hline Total & 145 & 27 & 32 & 35 & 23 & 16 & 7 & 5 \\
\hline
\end{tabular}

number of webpages that are returned, and from which users then need to choose. Analysis of the logfiles for the 23 occasions that date filtering was used showed that participants would have had to choose from an average of 152 pages (Internet Explorer) or 364 pages (Firefox), compared with 53 pages for our tool. Of course, this large reduction occurred because participants had to choose a specific date with our tool, but on 19 occasions they were correct first time, and on the other occasions participants made the correct choice at either the second or third attempt. Chrome paginates a user's history, so the longer ago a page was visited the greater the number of times the 'Older' button has to be clicked. However, once the required date has been located, a user would choose from the same number of pages as with our tool.

None of the browsers allows users to filter their history by both date and domain. Analysis of the logfiles for the 16 occasions that date/domain filtering was used showed that our tool narrowed participants' choice to an average of 6 webpages. By contrast, users would have needed to choose from an average of 71 (Chrome), 207 (Internet Explorer) or 264 pages (Firefox) if they had used a browser's date filtering functionality.

The searches filtering method displayed participants' search queries, search results that participants clicked, and any subsequent webpages to which participants browsed. This capability is not provided by any of the three browsers, but Google History ${ }^{10}$ does record search queries and search results that are clicked.

\subsubsection{Views and interface functionality for revisiting}

This section summarizes usage of the MyWebSteps list vs. tree view, and key interface functionality. In terms of the tool's views, 83 revisits involved the list view, 60 revisits involved the tree view, and two revisits involved both views. One participant explained: "I prefer the list view because its thumbnails were often much bigger than ones in tree. I just needed to scroll the list to find my wanted pages". Participants also suggested providing a grid view, which would display a large number of thumbnails in a compact format.

The key functionality was provided by the domains tab (used in 51 out of the 145 revisits), calendar (39 revisits), and the free text query dialog (27 revisits). Other functionality was less frequently used. For example, the searches tab and sessions tab were employed in only 12 and 4 revisits respectively. Buttons and filters on the toolbar were used in a total of 19 revisit sessions. Participants right clicked on a node in the tree to open the detailed dialog for further navigation in seven revisits.

\footnotetext{
${ }^{10}$ https://history.google.com/history
} 


\subsubsection{Reviewing}

During reviewing, a participant only looked at pages' thumbnails within the tool and did not use it to open any pages within the browser. The 92 reviewing sessions had an average duration of 40 seconds $(S D=83 s)$, but the longest was 9 minutes. To review their browsing history, participants most often selected a specific date ( $51 \%$ of sessions), with other common interaction patterns involving the selection of a domain $(12 \%)$, search queries $(9 \%)$ or topics $(9 \%)$.

Taking advantage of this reviewing capability, one participant commented:

"The tool made me aware of search habits and memory issues. Instead of typing in keywords, I often type in full questions. Also I sometimes look for the same things a month later without fully remembering that I have asked that question before."

Another participant highlighted new opportunities that the tool provided:

"I had a really amazing incidence where I could use your tool. I was spending the Saturday night in with a friend and we were watching a movie. We heard about something in the movie that was interesting and we did not know about so we started googling it. On that homepage (A) we found something $(B)$ that was super interesting as well so we searched for ' $B$ ' online, too. From $B$ we then came to $C$ and so on and so forth. We were basically rambling on all night. After literally hours of online searching we asked ourselves 'how did this happen, how/where did we start?' Obviously I had the answer to that question and opened your tool and showed the visualization screen to my friend. She was so amazed by the fact that we could trace our steps and that she could even see the little thumbnails. She was immediately asking where I got the tool from because she saw how useful and easy to handle it was. ...I can also see your tool as a tool for parents who want to see what their children are up to online all day."

\subsection{Discussion}

MyWebSteps was designed to help people revisit webpages that they had previously visited neither frequently nor recently - precisely the category of webpage for which today's Web browsers provide poor support. In fact, although half of the tool's usage was for this category of webpage, it was also used for other categories (see Table 5). The key reason was the advantage of recognition (based on page thumbnails), speed, and the small number of actions that participants needed to perform. These advantages led to participants preferring the tool to conventional Web browser history functionality such as auto-complete when directly entering a URL.

Half of the diary entries fell into the search results cause, highlighting how often participants preferred to browse/filter a visual history rather than try to find a webpage again using a search engine. The problems of revisiting webpages that users originally found via a search engine are well known (Capra and Pe-Quinones, 2005; Teevan et al., 2007), and the present study shows that visual tools such as MyWebSteps complement tools that are purpose-designed for repeat searches (e.g., (Teevan, 2007; Morris et al., 2008; Morgan and Wilson, 2010)).

The methods that participants used to revisit webpages revealed three key limitations of today's browsers. The first is that even when functionality is provided to filter history by domain that functionality is hidden (Internet Explorer; Chrome). By contrast, our tool presents users with a list of domains from which to select. Second, some browsers (e.g., Chrome) adopt an approach to filtering by date that scales poorly, because the number of clicks that are required to find a webpage that was visited on a given date increases linearly with the number of webpages that have been visited since that date. Third, browsers lack the ability to filter history precisely by both date and domain, leading to a 10 (Chrome) to 40 times increase (Firefox) in the number of history items that users need to choose from, compared with MyWebSteps.

As with the first evaluation (see Section 3), the small number and background of the participants means that some caution needs to be exercised when interpreting the results, and two particular points should be made. First, the very fact that that our participants volunteered for the evaluation means that they were probably receptive to using novel software applications, which may have contributed to the large percentage of participants who wished to continue using the tool. Second, a broader set of users would have a lower level of computer-literacy, meaning that they would be even less aware of the hidden functionality of today's browsers, exasperating the problems that were noted above.

Previous tools such as WebMap (Domel, 1995), PadPrints (Hightower et al., 1998), Domain Tree Browser (Gandhi et al., 2000) and SessionGraphs (Mayer and Bederson, 2001) have shown that visualization assists revisiting. However, MyWebSteps stands out for its capability to scale to the quantity of webpages that people visit over an extended period of time. This scalability is due to our tool recording all visits but only representing each page once in the visualization, the interface filters that allow user to rapidly reduce their whole history to a small set that concern a given domain/date/topic, and engineering the tool so that it is responsive even with large histories (in extended usage it performs well with an 18-month, 21,000 page history that includes $5 \mathrm{~Gb}$ of thumbnails).

In the field evaluation, participants used the research version of our tool that is described in Section 4. Since then we have publicly released three versions of the tool 
as the Firefox add-on MyWebSteps ${ }^{11}$. The remainder of this section summarizes the changes that have been made since the research version, which were informed by the results of the field evaluation and user feedback, and make other recommendations.

The first public version of MyWebSteps (Version 1.1.1) incorporated a number of changes. First, the field evaluation logfiles showed that participants rarely used some of the user interface functionality (Session tab, heat map legend in the Global Navigation area, Back/Forward and Month View buttons on the Toolbar, and filters for dwell time and number of days) so these were all removed to provide a simpler tool. A Grid View layout was added for viewing webpage thumbnails in the Result View, which improved scalability because a grid makes better use of display real estate than a tree layout. In addition, the Result View was changed so that it showed only one type of view at a time (List, Tree or Grid), and MyWebSteps was opened in a new tab of Firefox rather than a new window.

Soon after we released the first public version, Mozila removed the Firefox's status bar where icons of MyWebSteps were placed in. This required a new version of MyWebSteps (Version 1.2), which added the icons to the toolbar of Firefox. This highlights one of the challenges that researchers face when making their work widely available - the open-ended effort that is needed to maintain compatibility with new releases of third party tools. On another occasion, a user emailed us reporting that he had updated his browser and could not see webpage thumbnails. This turned out to be caused by Mozila changing the API for capturing a webpage's thumbnail, which we fixed with Version 1.3 of MyWebSteps.

Computers have widely differing amounts of disk space, so it is not surprising that some users like the benefits provided by storing a thumbnail of the whole of each webpage (this allows the original page to be read again) but another user complained about the amount of disk space that MyWebSteps took. Version 1.3 addresses that by only capturing the visible part of a webpage and, in the future, we may make that capture user-configurable.

A broader question is where to store history information (locally vs. in the cloud) and the trade-off that entails for privacy. During our research we prioritized privacy and stored the data locally, because web navigation activity is personal data. However, with the spread of cloud computing and high speed mobile internet, users are more likely to be willing to trade-off privacy for the convenience of being able to integrate their web history across all devices (e.g., computer, tablet and smart phone) and minimizing local storage needs.

\footnotetext{
${ }^{11}$ https://addons.mozilla.org/en-US/firefox/addon/mywebsteps/
}

Finally, there is some additional functionality that it would clearly be beneficial to provide. One is to support other search engines (this was not important for our research because all of our participants were Googleusers), and a second is to support other browsers as Firefox's market share has reduced since our research commenced. A third relates to webpages that are accessed via secure communication protocols. When the present research started, the https protocol was typically used for banking, online payments, and the submission of personal data. However, today https is much more widely used. Search engines such as Google, Yahoo and Bing now provide their search service via https, and one of our participants was surprised when he could not find in MyWebSteps any of the Coursera webpages that he had visited, they also use https. A solution would be to provide functionality for users to opt in to capturing https webpages from certain providers, to complement the opt out functionality that our tool has always provided for http webpages and domains.

\section{CONCLUSIONS}

Addressing the general topic of "keeping found things found", this research first investigated the underlying causes of failure when people tried to revisit webpages. Ten causes were identified by analyzing unsuccessful revisiting trials of a controlled laboratory experiment, data about participants' navigational actions during the experiment, video/audio of participants' thinking aloud and related data from participants' logfiles. The three main causes (accounting for $61 \%$ of the failures) were: (1) participants visiting a large number of pages on a particular topic, (2) webpages that had originally been accessed via search results, (3) participants knowing which website contained a page but that website itself being large. The second cause of failure can be explained by challenges of re-finding such as new ranking algorithms or updated databases (Aula et al., 2005; Teevan et al., 2007), recalling search queries, recognizing the search results on which users have clicked, and effectively browsing further from those pages (Obendorf et al., 2007).

Informed by these findings, and building on previous research, we designed and developed a novel visual Web history tool that supports both browsing and searching. Evidence about the benefits of MyWebSteps were provided through a three month field study in which participants succeeded during $96 \%$ of revisits. The study also revealed that participants also wanted to review their Web navigation history, to look up information they had lost, understand how they spent their time, or simply for fun. Reviewing activity might also enable people to improve their Web navigation strategy or support 
report/diary tasks, and highlights a use case for future history tools to consider. Following the success of the field study and participants' positive comments, we have publicly released MyWebSteps on the Firefox add-on center.

\section{ACKNOWLEDGEMENTS}

The first author was funded by a University of Leeds' Fully-Funded International Research Scholarship (FIRS). Thanks to members of the Visualization and Virtual Reality group for helpful comments made during our discussions. Special thanks to all the participants.

\section{REFERENCES}

Adar, E., Teevan, J. and Dumais, S.T. (2008) Large scale analysis of web revisitation patterns. In Proceedings of the SIGCHI Conference on Human Factors in Computing Systems, Florence, Italy, pp. 1197-1206. ACM, New York, NY, USA.

Aula, A., Jhaveri, N. and Kaki, M. (2005) Information search and re-access strategies of experienced web users. In Proceedings of the International Conference on World Wide Web, Chiba, Japan, pp. 583-592. ACM, New York, NY, USA.

Aula, A., Khan, R.M., Guan, Z., Fontes, P. and Hong, P. (2010) A comparison of visual and textual page previews in judging the helpfulness of web pages. In Proceedings of the International Conference on World Wide Web, Raleigh, North Carolina, USA, pp. 51-60. ACM, New York, NY, USA.

Badesh, H. and Blustein, J. (2012) VDMs for finding and re-finding web search results. In Proceedings of the 2012 iConference, Toronto, Ontario, Canada, pp. 419-420. ACM, New York, NY, USA.

Breslau, L., Pei, C., Li, F., Phillips, G. and Shenker, S. (1999) Web caching and Zipf-like distributions: evidence and implications. In Proceedings of the Eighteenth Annual Joint Conference of the IEEE Computer and Communications Societies (IEEE INFOCOM '99), pp. 126-134. IEEE, Washington DC, USA.

Bruce, H., Jones, W. and Dumais, S. (2004) Keeping and refinding Information on the Web: What do people do and what do they need? In Proceedings of the American Society for Information Science and Technology, Providence, Rhode Island, USA, pp. 129-137. American Society for Information Science and Technology.

Capra, R.G. and Perez-Quinones, M.A. (2005) Using Web search engines to find and refind information. Computer, 38, $36-42$.

Catledge, L.D. and Pitkow, J.E. (1995) Characterizing browsing strategies in the World-Wide Web. Computer Networks and ISDN Systems, 27, 1065-1073.

Ceri, S., Daniel, F., Matera, M. and Rizzo, F. (2006) Extended memory (xMem) of web interactions. In Proceedings of the International Conference on Web Engineering, Palo Alto, California, USA, pp. 177 - 184. ACM, New York, NY, USA.

Cockburn, A. and Jones, S. (1996) Which way now? Analysing and easing inadequacies in WWW navigation. International Journal of Human-Computer Studies, 45, 105-129.

Cockburn, A. and Mckenzie, B. (2001) 3D or not 3D? Evaluating the effect of the third dimension in a document management system. In Proceedings of the SIGCHI Conference on Human Factors in Computing Systems, pp. 434-441. ACM, New York, NY, USA.

Cockburn, A. and Mckenzie, B. (2001) What do web users do? An empirical analysis of web use. International Journal of Human-Computer Studies, 54, 903-922.

Cohen, J. (1960) A coefficient of agreement for nominal scales. Educational and Psychological Measurement, 20, 37- 46.

Dai, N. and Davison, B.D. (2010) Mining anchor text trends for retrieval. In Proceedings of the European Conference on Information Retrieval, Milton Keynes, UK, pp. 127-139. Springer, Berlin, Heidelberg.

Dijkstra, E.W. (1959) A note on two problems in connexion with graphs. Numerische Mathematik, 1, 269-271.

Domel, P. (1995) WebMap: a graphical hypertext navigation tool. Computer Networks and ISDN Systems, 28, 85-97.

Dziadosz, S. and Chandrasekar, R. (2002) Do thumbnail previews help users make better relevance decisions about web search results? In Proceedings of the International Conference on World Wide Web, Tampere, Finland, pp. 365366. ACM, New York, NY, USA.

Eklund, J., Sawers, J. and Zeiliger, R. (1999) NESTOR navigator: a tool for the collaborative construction of knowledge through constructive navigation. In Proceedings of the Australian World Wide Web Conference, Ballina, NSW, Australia, pp. 396-408.

Elsweiler, D., Baillie, M. and Ruthven, I. (2011) What makes re-finding information difficult? A study of email re-finding. In Proceedings of the European Conference on Information Retrieval, Dublin, Ireland, pp. 568-579. Springer-Verlag, Heidelberg.

Elsweiler, D. and Ruthven, I. (2007) Towards task-based personal information management evaluations. In Proceedings of the International ACM SIGIR Conference on Research and Development in Information Retrieval, Amsterdam, The Netherlands, pp. 23-30. ACM New York, NY, USA.

Erlandson, D.A., Harris, E.L., Skipper, B.L. and Allen, S.D. (1993) Doing Naturalistic Inquiry: A Guide to Methods. Sage.

Feldman, S. (2004) The high cost of not finding information. KMWorld, 13.

Fox, S., Karnawat, K., Mydland, M., Dumais, S. and White, T. (2005) Evaluating implicit measures to improve web search. ACM Transactions on Information Systems, 23, 147-168. 
Frecon, E. and Smith, G. (1998) WebPath - A ThreeDimensional Web History. In Proceedings of the IEEE Symposium on Information Visualization, North Carolina, pp. 3-10. IEEE Computer Society, Washington, DC, USA.

Fujii, A. (2008) Modeling anchor text and classifying queries to enhance web document retrieval. In Proceedings of the International Conference on World Wide Web, Beijing, China, pp. 337-346. ACM, New York, NY, USA.

Furnas, G.W. (1986) Generalized fisheye views. In Proceedings of the SIGCHI Conference on Human Factors in Computing Systems, Boston, Massachusetts, USA, pp. 16-23. ACM, New York, NY, USA.

Gandhi, R., Kumar, G., Bederson, B. and Shneiderman, B. (2000) Domain name based visualization of web histories in a zoomable user interface. In Proceedings of the International Workshop on Database and Expert Systems Applications, Greenwich, London, United Kingdom, pp. 591-598. IEEE Computer Society, Washington, DC, USA.

Gwizdka, J. and Spence, I. (2007) Implicit measures of lostness and success in web navigation. Interacting with Computers, 19, 357-369.

Haney, W., Russell, M., Gulek, C. and Fierros, E. (1998) Drawing on education: Using student drawings to promote middle school improvement. Schools in the Middle, 7, 38- 43.

Hightower, R.R., Ring, L.T., Helfman, J.I., Bederson, B.B. and Hollan, J.D. (1998) Graphical multiscale Web histories: a study of padprints. In Proceedings of the ACM Conference on Hypertext and Hypermedia, Pittsburgh, Pennsylvania, United States, pp. 58 - 65. ACM, New York, NY, USA.

Jiao, B., Yang, L., Xu, J. and Wu, F. (2010) Visual summarization of web pages. In Proceedings of the International ACM SIGIR Conference on Research and Development in Information Retrieval, Geneva, Switzerland, pp. 499-506. ACM, New York, NY, USA.

Jones, W., Bruce, H. and Dumais, S. (2001) Keeping found things found on the web. In Proceedings of the International Conference on Information and Knowledge Management, Atlanta, Georgia, USA, pp. 119 - 126. ACM, New York, NY, USA.

Jones, W., Bruce, H. and Dumais, S. (2003) How do people get back to information on the web? How can they do it better. In Proceedings of INTERACT, pp. 793-796.

Jones, W., Capra, R., Diekema, A., Teevan, J., PerezQuinones, M., Dinneen, J.D. and Hemminger, B. (2015) "For Telling" the Present: Using the Delphi Method to Understand Personal Information Management Practices. In Proceedings of the SIGCHI Conference on Human Factors in Computing Systems, Seoul, Republic of Korea, pp. 35133522. ACM, New York, NY, USA.

Kaasten, S., Greenberg, S. and Edwards, C. (2002) How people recognize previously seen Web pages from titles, URLs and thumbnails. In Proceedings of BCS Human Computer Interaction, London, UK, pp. 247-265. British Computer Society, Swinton, UK.
Kelly, D. and Belkin, N.J. (2004) Display time as implicit feedback: understanding task effects. In Proceedings of the International ACM SIGIR Conference on Research and Development in Information Retrieval, Sheffield, United Kingdom, pp. 377 - 384. ACM, New York, NY, USA.

Kokosis, P., Krikos, V., Stamou, S. and Christodoulakis, D. (2005) HiBO: a system for automatically organizing bookmarks. In Proceedings of the ACM/IEEE-CS joint Conference on Digital Libraries, Denver, Colorado, USA, pp. 155-156. ACM, New York, NY, USA.

Koolen, M. and Kamps, J. (2010) The importance of anchor text for ad hoc search revisited. In Proceedings of the International ACM SIGIR Conference on Research and Development in Information Retrieval, Geneva, Switzerland, pp. 122-129. ACM, New York, NY, USA.

Li, J. and Zhao, Y. (2009) PathRank: Web page retrieval with navigation path. In Proceedings of the European Conference on Information Retrieval, Toulouse, France, pp. 350-361. Springer, Berlin, Heidelberg.

Liu, C., White, R.W. and Dumais, S. (2010) Understanding web browsing behaviors through Weibull analysis of dwell time. In Proceedings of the International ACM SIGIR Conference on Research and Development in Information Retrieval, Geneva, Switzerland, pp. 379-386. ACM, New York, NY, USA.

Loumakis, F., Stumpf, S. and Grayson, D. (2011) This image smells good: effects of image information scent in search engine results pages. In Proceedings of the International Conference on Information and Knowledge Management, Glasgow, Scotland, UK, pp. 475-484. ACM, New York, NY, USA.

Mayer, M. (2009) Web history tools and revisitation support: a survey of existing approaches and directions. Foundations and Trends in Human-Computer Interaction, 2, 173-278.

Mayer, M. and Bederson, B.B. (2001) Browsing icons: a taskbased approach for a visual Web history. In HCIL Technical Report, CS-TR-4308, MD, USA. University of Maryland.

Miller, F.P., Vandome, A.F. and Mcbrewster, J. (2010) Delicious (website). Alpha Press.

Morgan, R. and Wilson, M.L. (2010) The Revisit Rack: grouping web search thumbnails for optimal visual recognition. In The Annual Meeting of the American Society for Information Science and Technology, Pittsburgh, Pennsylvania, pp. 1-4. American Society for Information Science.

Morris, D., Morris, M.R. and Venolia, G. (2008) SearchBar: a search-centric web history for task resumption and information re-finding. In Proceedings of the SIGCHI Conference on Human Factors in Computing Systems, Florence, Italy, pp. 1207-1216. ACM, New York, NY, USA.

Ntoulas, A., Cho, J. and Olston, C. (2004) What's new on the web?: the evolution of the web from a search engine perspective. In Proceedings of the International Conference on World Wide Web, pp. 1-12. ACM, New York, NY, USA.

Obendorf, H., Weinreich, H., Herder, E. and Mayer, M. (2007) 
Web page revisitation revisited: implications of a longterm click-stream study of browser usage. In Proceedings of the SIGCHI Conference on Human Factors in Computing Systems, San Jose, California, USA, pp. 597 - 606. ACM, New York, NY, USA.

Panasiti, G. (2009) Display your browsing history as a tree: History Tree. Retrieved 20 February 2010, from http://www.browserland.com/add-ons/display-yourbrowsing-history-as-a-tree-history-tree/.

Robertson, G., Czerwinski, M., Larson, K., Robbins, D.C., Thiel, D. and Dantzich, M.V. (1998) Data mountain: using spatial memory for document management. In Proceedings of the ACM Symposium on User Interface Software and Technology, San Francisco, California, United States, pp. 153 - 162. ACM, New York, NY, USA.

Staff, C. and Bugeja, I. (2007) Automatic classification of web pages into bookmark categories. In Proceedings of the International ACM SIGIR Conference on Research and Development in Information Retrieval, Amsterdam, The Netherlands, pp. 731-732. ACM, New York, NY, USA.

Tauscher, L. and Greenberg, S. (1997) How people revisit web pages: empirical findings and implications for the design of history systems. International Journal of Human-Computer Studies, 47, 97-137.

Teevan, J. (2007) The re:search engine: simultaneous support for finding and re-finding. In Proceedings of the ACM Symposium on User Interface Software and Technology, Newport, Rhode Island, USA, pp. 23-32. ACM, New York, NY, USA.

Teevan, J., Adar, E., Jones, R. and Potts, M.a.S. (2007) Information re-retrieval: repeat queries in Yahoo's logs. In Proceedings of the International ACM SIGIR Conference on Research and Development in Information Retrieval, Amsterdam, The Netherlands, pp. 151 - 158. ACM, New York, NY, USA.

Teevan, J., Alvarado, C., Ackerman, M.S. and Karger, D.R. (2004) The perfect search engine is not enough: a study of orienteering behavior in directed search. In Proceedings of the SIGCHI Conference on Human Factors in Computing Systems, Vienna, Austria, pp. 415 - 422. ACM, New York, NY, USA.

Teevan, J., Cutrell, E., Fisher, D., Drucker, S.M., Ramos, G., Andre, P. and $\mathrm{Hu}, \mathrm{C}$. (2009) Visual snippets: summarizing web pages for search and revisitation. In Proceedings of the SIGCHI Conference on Human Factors in Computing Systems, Boston, MA, USA, pp. 2023-2032. ACM, New York, NY.

Tunkelang, D. (2009) Faceted Search. Morgan and Claypool Publishers.

Tyler, S.K. and Teevan, J. (2010) Large scale query log analysis of re-finding. In Proceedings of the ACM International Conference on Web Search and Data Mining, New York, New York, USA, pp. 191-200. ACM, New York, NY, USA.

Walker, J.Q. (1990) A node-positioning algorithm for general trees. Software - Practice \& Experience, 20(7), 685-705.

Weber, R.P. (1990) Basic Content Analysis. Sage.

Weinreich, H., Obendorf, H., Herder, E. and Mayer, M. (2006) Off the beaten tracks: exploring three aspects of web navigation. In Proceedings of the International Conference on World Wide Web, Edinburgh, Scotland, pp. 133 - 142. ACM, New York, NY, USA.

Wexelblat, A. and Maes, P. (1999) Footprints: history-rich tools for information foraging. In Proceedings of the SIGCHI Conference on Human Factors in Computing Systems, Pittsburgh, Pennsylvania, United States, pp. 270 - 277. ACM, New York, NY, USA.

White, R.W. and Huang, J. (2010) Assessing the scenic route: measuring the value of search trails in web logs. In Proceedings of the International ACM SIGIR Conference on Research and Development in Information Retrieval, Geneva, Switzerland, pp. 587-594. ACM, New York, NY, USA.

Won, S.S., Jin, J. and Hong, J.I. (2009) Contextual web history: using visual and contextual cues to improve web browser history. In Proceedings of the SIGCHI Conference on Human Factors in Computing Systems, Boston, MA, USA, pp. 1457-1466. ACM, New York, NY, USA.

Woodruff, A., Faulring, A., Rosenholtz, R., Morrsion, J. and Pirolli, P. (2001) Using thumbnails to search the Web. In Proceedings of the SIGCHI Conference on Human Factors in Computing Systems, Seattle, Washington, USA, pp. 198205. ACM, New York, NY, USA.

Woodruff, A., Rosenholtz, R., Morrison, J.B., Faulring, A. and Pirolli, P. (2002) A comparison of the use of text summaries, plain thumbnails, and enhanced thumbnails for Web search tasks. Journal of the American Society for Information Science and Technology, 53, 172-185.

Zhang, H. and Zhao, S. (2011) Measuring web page revisitation in tabbed browsing. In Proceedings of the SIGCHI Conference on Human Factors in Computing Systems, Vancouver, BC, Canada, pp. 1831-1834. ACM, New York, NY, USA. 